\title{
THE MOTIVIC IGUSA ZETA SERIES OF SOME HYPERSURFACES NON-DEGENERATED WITH RESPECT TO THEIR NEWTON POLYHEDRON
}

\author{
Hans Schoutens
}

\begin{abstract}
We describe some algorithms, without using resolution of singularities, that establish the rationality of the motivic Igusa zeta series of certain hypersurfaces that are non-degenerated with respect to their Newton polyhedron. This includes, in any characteristic, the motivic rationality for polydiagonal hypersurfaces, vertex singularities, binomial hypersurfaces, and Du Val singularities.
\end{abstract}

\section{Introduction}

Fix a field $\kappa$, of arbitrary characteristic, but often also assumed to be algebraically closed. We use the term scheme $W$ to mean a separated scheme of finite type over $\kappa$; and variety to mean a reduced scheme (so that varieties are not assumed to be irreducible). Denef and Loeser have proposed a motivic variant of the classical Igusa zeta series ([3, 12]), and showed its rationality in characteristic zero by means of motivic integration, quantifier elimination for Henselian valued fields, and resolution of singularities (the latter two are only available at present in characteristic zero, whence their restriction to this case; see [5, 7]). Recall that the Grothendieck ring $\mathfrak{G} \mathfrak{r}\left(\operatorname{Var}_{\kappa}\right)$ is the universal Euler characteristic on varieties over $\kappa$, that is to say, the quotient of the free Abelian group on isomorphism classes $[V]$ of varieties $V$ over $\kappa$ modulo the

Received: 14.04.2016. Accepted: 25.06.2016.

(2010) Mathematics Subject Classification: 14G10,18F30.

Key words and phrases: motivic Igusa zeta series, Du Val singularities, stationary phase method. 
scissor relations

$$
[V]=[V-W]+[W]
$$

for $W$ a locally closed subvariety of $V$, with multiplication given by fiber products. For each $n$, let $L_{n}(W)$ be the $n$-th truncated jet space of $W$ (see below for a discussion), and put

$$
\operatorname{Igu}_{W}(t):=\sum_{n=1}^{\infty}\left[L_{n}(W)\right] t^{n}
$$

as a power series over $\mathfrak{G} \mathfrak{r}\left(\operatorname{Var}_{\kappa}\right)$. They prove in [5] that $\operatorname{Ig}_{W}$ is rational over the localization $\mathfrak{G}:=\mathfrak{G r}\left(\operatorname{Var}_{\kappa}\right)_{\mathbb{L}}$, where $\mathbb{L}:=\left[\mathbb{A}_{\kappa}^{1}\right]$ denotes the Lefschetz class. Moreover, they also show that this motivic Igusa zeta series specializes to the classical one-thus reproving its rationality, but gaining in the process an enormous amount of uniformity-as follows: if $W_{0}$ is a model of $W$ over a finitely generated $\mathbb{Z}$-algebra $\mathcal{O}$, then for almost all maximal ideals $\mathfrak{m} \subseteq \mathcal{O}$, the motivic Igusa zeta series specializes to the classical one via the counting function $\left[L_{n}(W)\right] \mapsto\left|W_{0}\left(\mathcal{O} / \mathfrak{m}^{n}\right)\right|$.

In the present paper, we will show rationality, in arbitrary characteristic, for a class of hypersurfaces $W \subseteq \mathbb{A}_{\kappa}^{d+1}$. In fact, we will describe a very explicit algorithm that allows one to calculate the motivic Igusa zeta series. It should be mentioned from the onset that this is done not via resolution of singularities ${ }^{2}$ and hence not in the usual terms of exceptional divisors. In fact, the main motivation for the present work was to get a different approach to motivic rationality, avoiding resolution of singularities, not only so that we would also obtain results in positive characteristic, but also in order to get more explicit formulas: the method using resolution, going back to work of Igusa and Denef, calculates poles in terms of the dual graph of the resolution, but it is a well-known and irritating fact that the resulting rational function has far too many apparent poles. This is the main stumble-block for verifying the monodromy conjecture $([19,20]$ ), and has resulted in an extensive literature about locating 'candidate poles' (see [21] and the references therein). As all worked-out examples so far show, this phenomenon does not occur in our approach.

To discuss the algorithm-which is a motivic variant of a method introduced by Igusa, which he coined the stationary phase method; see [11, 15] and the references therein-let me restrict here to the case of a plane curve in

\footnotetext{
${ }^{1}$ Different indexing practices might lead to slightly different series as found in the literature.

${ }^{2}$ Indeed, all rationality results in this paper can be proven, regardless of characteristic, by this method, since hypersurfaces that are non-degenerate with respect to their Newton polyhedron admit embedded resolutions by toric methods.
} 
characteristic zero, given by a polynomial $F(X, Y)$ with a unique singularity at the origin $O$. To a variable $X$, we associate in general a generic jet

$$
\widehat{x}:=x_{0}+x_{1} \xi+x_{2} \xi^{2}+\ldots
$$

in the countable new parametric variables $x_{i}$, called the jet variables. Here is how we calculate $\nabla_{n} W$, for a given $n$. The equations of $\nabla_{n} W$ are the coefficients-given by some polynomials in the first $n$ jet variables, and called the jet equations-of the first $n$ powers of $\xi$ in the expansion of $F(\widehat{x}, \widehat{y})$.

In its simplest form, the algorithm is encoded by a tree, where each time a splitting occurs between smooth and non-smooth loci (see Corollary 2.2). This is motivated by the fact that $\rho: \nabla_{n} W \rightarrow W$ is a locally trivial fibration on the smooth locus of $W$, that is to say, away from the origin, so that the branch over the smooth locus is easy, and the only complications arise above the origin. The jet fiber $\rho^{-1}(O)$ above the origin is obtained by putting $x_{0}=y_{0}=0$ in the jet equations. After this substitution, we can factor out some power $\xi^{e}$ from $F(\widehat{x}, \widehat{y})$. The sum $\tilde{F}$ of the monomials in $F$ which have lowest order in this new expansion is called the first initial form of $F$. If $\tilde{F}$ has again a singularity at the origin, so that the new jet fiber above the singularity is obtained by putting $x_{1}=y_{1}=0$, we can repeat the process to the curve given by the initial form $\tilde{F}$, where, because of the extra factor $\xi^{e}$, we now only have to consider jets of length $n-e$. However, it may happen that $\tilde{F}$ is just a monomial, the smooth locus of which is empty. In this case, we formally invert one of the jet variables, and only put the remaining variables to zero. We show that all but one of the leafs (=endpoints) in this tree come from some smooth curve, with the remaining one, after a finite number of steps, yielding jet equations which are isomorphic to the original ones (or, more generally, to one of the previously found jet equations). This yields a recursion relation among different jet spaces, from which the rationality of $\operatorname{Igu}_{W}$ then easily follows.

Let me illustrate this on the cuspidal curve $F=X^{2}-Y^{3}$. The jet equations are given by the expansion

$$
\left(x_{0}+x_{1} \xi+\ldots\right)^{2}-\left(y_{0}+y_{1} \xi+\ldots\right)^{3}=\left(x_{0}^{2}-y_{0}^{3}\right)+\left(2 x_{0} x_{1}-3 y_{0}^{2} y_{1}\right) \xi+\ldots
$$

as the coefficients of the powers $1, \xi, \ldots, \xi^{n-1}$. Let us write

$$
\widetilde{x(i)}:=x_{i}+x_{i+1} \xi+x_{i+2} \xi^{2}+\ldots
$$

for the $i$-th twisted generic jet. Putting $x_{0}$ and $y_{0}$ equal to zero, the jet fiber above the origin is then given by

$$
\left(x_{1} \xi+\ldots\right)^{2}-\left(y_{1} \xi+\ldots\right)^{3}=\xi^{2}\left(\widetilde{x(1)}^{2}-\widetilde{y(1)}^{3} \xi\right)
$$


showing that $\tilde{F}=X^{2}$. The singular locus of $\tilde{F}$ is therefore given by $x_{1}=0$ (the smooth locus is empty, yielding a "dead" branch in the algorithm tree), and the jet fiber above this locus is given by

$$
\left(x_{2} \xi^{2}+\ldots\right)^{2}-\left(y_{1} \xi+\ldots\right)^{3}=\xi^{3}\left(\widetilde{x(2)}^{2} \xi-\widetilde{y(1)}^{3}\right) .
$$

This time $Y^{3}$ is the initial form, forcing us to consider its singular locus $y_{1}=0$. This in turn gives again the initial form $X^{2}$, prompting the additional equation $x_{2}=0$, so that we finally arrive at

$$
\left(x_{3} \xi^{3}+x_{4} \xi^{4}+\ldots\right)^{2}-\left(y_{2} \xi^{2}+y_{3} \xi^{3}+\ldots\right)^{3}=\xi^{6}\left(\overline{x(3)}^{2}-\overline{y(2}^{3}\right) .
$$

Factoring out $\xi^{6}$, we see that we get, up to the change of variables $x_{i} \mapsto x_{i-3}$ and $y_{i} \mapsto y_{i-2}$, the same expansion as in (1.1), except that we now only have to look at the first $n-6$ terms. The former therefore leads to a recursion relation between $\left[\nabla_{n} W\right]$ and $\left[\nabla_{n-6} W\right]$, and, ultimately, to a relation between $\operatorname{Igu}_{W}$ and $t^{6} \operatorname{Igu}_{W}$, so that, when solved for $\operatorname{Igu}_{W}$, proves the latter's rationality.

The main point of the algorithm therefore is that at each point, we can make the initial form non-singular by inverting some of the variables. In the literature, this phenomenon is referred to as being non-degenerate with respect to its Newton polyhedron (see, for instance, [13, 15]). I expect that our rationalization algorithm can be made to work for any such hypersurface, but at present, some extra control of the growth of various monomials is required, and we will establish this only for the following types:

diagonal given by an equation $u_{0} X_{0}^{n_{0}}+\cdots+u_{d} X_{d}^{n_{d}}=0$ with an isolated singularity (the latter condition holds automatically in characteristic zero and holds in characteristic $p$ if at most one of the $n_{i}$ is divisible by $p$; see Theorem 5.9);

vertex singularity given by the cone on a smooth projective variety, or analytically isomorphic to such a cone; see $\$$.

Du Val singularity or canonical isolated surface singularity; see $\$ 6$. linear deformation of a poly-diagonal given by an equation of the form

$$
u_{1} X_{1}^{n_{1}}+\cdots+u_{d} X_{d}^{n_{d}}+v_{1} X_{j(1)} \mathbf{Y}_{1}^{\mu_{1}}+\cdots+v_{e} X_{j(e)} \mathbf{Y}_{e}^{\mu_{e}}=0
$$

where $\left(X_{1}, \ldots, X_{d}\right)$ and the $\mathbf{Y}_{i}$ are tuples of distinct variables.

Most of these cases are subsumed under the latter case, where in positive characteristic, we have to impose some separability condition; see Theorem 7.3 . We should add that similar results are obtained in [1, Theorem 2.4] and [9, Proposition 2.1.3] using methods from toric resolution, similar to the $p$-adic case from [4]. Whereas these results apply to the class of all non-degenerated hypersurfaces, the poles of the resulting rational functions seem harder to 
analyze. The stationary phase method is also applied to non-degenerate hypersurfaces in [22, 23], though not in the motivic setting.

\section{Jet schemes}

We work over a fixed algebraically closed field $\kappa$, and 'scheme' will always means separated scheme of finite type over $\kappa$. The $n$-th jet scheme of a scheme $W$, denoted $\nabla_{n} W$, is by definition the Hilbert scheme of $\kappa[\xi] / \xi^{n} \kappa[\xi]$-rational points, that is to say

$$
\operatorname{Mor}_{\kappa}\left(\operatorname{Spec}\left(A[\xi] / \xi^{n} A[\xi]\right), W\right) \cong \operatorname{Mor}_{\kappa}\left(\operatorname{Spec} A, \nabla_{n} W\right),
$$

for any $\kappa$-algebra $A$. Taking $A=\kappa$, we recover the $n$-th truncated jet space $L_{n}(W)$ as the reduction of $\nabla_{n} W$. Below we will describe a general method to calculate these jet schemes, from which their existence and uniqueness can be inferred (for some rigorous proof see [2] or for a more general version, see [18, §4]). An important point has to be borne in mind: in contrast with Denef and Loeser, we calculate jet schemes of arbitrary schemes, not just varieties. Note that taking jets does not commute with taking reduction. The present method is, in fact, inspired by my work on schemic Grothendieck rings [17, 18] (in which Grothendieck rings are proposed that can handle nilpotent structure), although in the present paper, we will only take classes of jets inside the classical Grothendieck ring, thus disregarding their nilpotent structure. I do not know whether the motivic Igusa zeta series are already rational over these more general schemic Grothendieck rings. The main point in allowing non-reduced schemes, however, is that, even when primarily interested in a reduced hypersurface in this paper, we must consider certain initial forms that are not necessarily reduced in order for the algorithm to work.

We let $\rho_{W, n}: \nabla_{n} W \rightarrow W$ be the canonical split projection (associating to a jet its origin) with section $W \hookrightarrow \nabla_{n} W$, and we view closed subschemes of $W$ as closed subschemes of $\nabla_{n} W$ via the latter embedding. Recall the following fibration theorem (see, for instance, [6, Lemma 4.1] or [18, Theorem 4.14]).

THEOREM 2.1. If $W$ is a d-dimensional smooth variety, then the split projection $\rho_{W, n}: \nabla_{n} W \rightarrow W$ is a locally trivial fibration with general fiber $\mathbb{A}_{\kappa}^{d(n-1)}$. In particular,

$$
\left[\nabla_{n} W\right]=[W] \cdot \mathbb{L}^{d(n-1)}
$$

in $\mathfrak{G} \mathfrak{r}\left(\operatorname{Var}_{\kappa}\right)$. 
COROLlary 2.2. Given a d-dimensional scheme $W$, and a closed subscheme $V$ containing the singular locus of $W$, we have an equality

$$
\left[\nabla_{n} W\right]=[W-V] \cdot \mathbb{L}^{d(n-1)}+\left[\rho_{W, n}^{-1}(V)\right]
$$

in $\mathfrak{G r}\left(\operatorname{Var}_{\kappa}\right)$.

Proof. Let $\rho:=\rho_{W, n}$. Put $W^{\prime}:=\nabla_{n} W$ and $V^{\prime}:=\rho^{-1}(V)$, so that

$$
\left[W^{\prime}\right]=\left[W^{\prime}-V^{\prime}\right]+\left[V^{\prime}\right]
$$

in $\mathfrak{G r}\left(\operatorname{Var}_{\kappa}\right)$. By the openness of jets see, for instance, [18, Theorem 4.4]), we have an isomorphism $W^{\prime}-V^{\prime} \cong \nabla_{n}(W-V)$. Since $W-V$ is smooth by the choice of $V$, Theorem 2.1 yields $\left[\nabla_{n}(W-V)\right]=[W-V] \cdot \mathbb{L}^{d(n-1)}$, and the assertion follows.

Given two closed immersions $W \hookrightarrow V$ and $Y \hookrightarrow X$, we say that their analytic germs are isomorphic, if the formal completions $\widehat{V}_{W}$ and $\widehat{X}_{Y}$ are isomorphic as formal schemes (for formal schemes, see [10, II.§9]). In case $W=P$ and $Y=Q$ are closed points, this means that the completions of the local rings $\mathcal{O}_{V, P}$ and $\mathcal{O}_{X, Q}$ are isomorphic (or, in the terminology of [16], these local rings are similar).

Proposition 2.3. If the closed immersions $W \hookrightarrow V$ and $Y \hookrightarrow X$ have isomorphic analytic germs, then

$$
\left[\rho_{V, n}^{-1}(W)\right]=\left[\rho_{X, n}^{-1}(Y)\right]
$$

in $\mathfrak{G} \mathfrak{r}\left(\operatorname{Var}_{\kappa}\right)$.

Proof. One could prove this directly, but it readily follows from the formalism from [18]. By [18, Lemma 4.9], we have, for each pair, an isomorphism of formal motives

$$
\nabla_{n}\left(\widehat{V}_{W}\right) \cong\left(\widehat{\nabla_{n} V}\right)_{\rho_{V, n}^{-1}(W)} \text { and } \quad \nabla_{n}\left(\widehat{X}_{Y}\right) \cong\left(\widehat{\nabla_{n} X}\right)_{\rho_{X, n}^{-1}(Y)},
$$

By assumption, $\widehat{V}_{W} \cong \widehat{X}_{Y}$, and this isomorphism is preserved after taking jets, and so we get

$$
\left(\widehat{\nabla_{n} V}\right)_{\rho_{V, n}^{-1}(W)} \cong\left(\widehat{\nabla_{n} X}\right)_{\rho_{X, n}^{-1}(Y)} .
$$

Taking classes yields an equality in the formal Grothendieck ring, whence one in the classical Grothendieck ring using [17, Theorem 7.7]. In other words, 
the underlying reduced varieties have the same class in $\mathfrak{G r}\left(\operatorname{Var}_{\kappa}\right)$. Since the underlying variety of a formal completion is just the locus along which one completes, we get the desired equality.

Let us define the analytic singularities of a variety $W$ as its formal completion $\widehat{W}_{W^{\text {sing }}}$ along its singular locus $W^{\text {sing }}$.

COROLlary 2.4. Let $V$ and $W$ be varieties with isomorphic analytic singularities. Then $\operatorname{Igu}_{V}$ is rational over $\mathfrak{G}$ if and only if $\operatorname{Igu}_{W}$ is.

Proof. Let $d$ and $e$ be the respective dimensions of $V$ and $W$. By Corollary 2.2, we have, for each $n$, an equality

$$
\begin{gathered}
{\left[\nabla_{n} V\right]=\left[V-V^{\text {sing }}\right] \cdot \mathbb{L}^{d(n-1)}+\left[\rho_{V, n}^{-1}\left(V^{\text {sing }}\right)\right]} \\
{\left[\nabla_{n} W\right]=\left[W-W^{\text {sing }}\right] \cdot \mathbb{L}^{e(n-1)}+\left[\rho_{W, n}^{-1}\left(W^{\text {sing }}\right)\right]}
\end{gathered}
$$

in $\mathfrak{G} \mathfrak{r}\left(\operatorname{Var}_{\kappa}\right)$. Moreover, by our assumption and Proposition 2.3, we also have

$$
\left[\rho_{V, n}^{-1}\left(V^{\text {sing }}\right)\right]=\left[\rho_{W, n}^{-1}\left(W^{\text {sing }}\right)\right] .
$$

Multiplying all terms in (2.1) by $t^{n}$, summing over all $n$, and using (2.2) then yields

$$
\operatorname{Igu}_{V}-\frac{\mathbb{L}^{d}\left[V-V^{\text {sing }}\right]}{1-\mathbb{L}^{d}}=\operatorname{Igu}_{W}-\frac{\mathbb{L}^{e}\left[W-W^{\text {sing }}\right]}{1-\mathbb{L}^{e}}
$$

over $\mathfrak{G}$, from which the assertion is now immediate.

\section{Directed jets}

In this section, we fix the formalism needed to describe our rationalization algorithms.

\section{Tagged and formal equations}

To any natural number $a$, we associate its tagged version $a^{\sharp}$, and we call $a$ the underlying value (or untagged version) of $a^{\sharp}$, which we might denote for emphasis by $a^{\natural}$. We can add tagged and/or untagged numbers by the rule that 
the underlying value of the sum is the sum of the underlying values of the terms, where the sum is tagged if and only if at least one term is tagged (e.g,. $2+3^{\sharp}=5^{\sharp}$ ). Let $\mathbb{N}_{\sharp}$ be the collection of all natural numbers and their tagged versions. We extend this component-wise on tuples giving a map $\mathbb{N}_{\sharp}^{m} \rightarrow \mathbb{N}^{m}$, sending a tuple $\boldsymbol{\theta} \in \mathbb{N}_{\sharp}^{m}$ to its underlying value $\boldsymbol{\theta}^{\natural}$. We define a partial order on $\mathbb{N}_{\sharp}^{m}$ by $\boldsymbol{\alpha} \leq \boldsymbol{\beta}$ if and only if for each $j=1, \ldots, m$, either $\alpha_{j}$ is untagged and $\alpha_{j} \leqslant \beta_{j}^{\natural}$, or $\alpha_{j}$ is tagged and $\alpha_{j}=\beta_{j}$.

We will introduce two equational conventions in this section that are useful for discussing jet equations. To each variable $X$ (we use uppercase letters to indicate indeterminates defining the base schemes), we associate its tagged version $X_{\sharp}$, which we will treat as an invertible variable. Given a tagged number $a^{\sharp}$, we write

$$
X_{\sharp}^{a}:=X^{a^{\sharp}}=X_{\sharp}^{a^{\sharp}} .
$$

Hence, we may associate to a polynomial $F \in \kappa[X]$, the polynomial $F\left(X^{\sharp}\right)$, which is just $F(X)$ but viewed in the Laurent polynomial ring $\kappa\left[X, \frac{1}{X}\right]$. Therefore, we interpret the equation $F\left(X^{\sharp}\right)=0$ as defining the locally closed subscheme given by $F(X)=0$ and $X$ is invertible. We may extend this practice to several variables, tagging some and leaving others unchanged. For instance, the tagged equation $X_{\sharp}^{2}+X_{\sharp} Y^{3}+Z_{\sharp}^{3}=0$ should be thought of as an element of the mixed Laurent polynomial ring $\kappa\left[X, Y, Z, \frac{1}{X}, \frac{1}{Z}\right]$, and is equivalent with the conditions $X^{2}+X Y^{3}+Z^{3}=0$ together with the requirement that $X$ and $Z$ are invertible. In particular, in a given polynomial, if a variable is tagged somewhere, it must be so everywhere. More precisely, for $\boldsymbol{\theta} \in \mathbb{N}_{\sharp}^{m}$, we call a variable $Y_{i}$ in $\mathbf{Y}$ tagged by $\boldsymbol{\theta}$, if the corresponding entry $\theta_{i}$ in $\boldsymbol{\theta}$ is tagged, and we refer to $\theta_{i}$ as the weight of $Y_{i}$. Let $\kappa\left\{\mathbf{Y}^{\boldsymbol{\theta}}\right\}$ be the algebra generated over the polynomial ring $\kappa[\mathbf{Y}]$ by the the inverses of the variables that are tagged by $\boldsymbol{\theta}$; we refer to such an algebra as a mixed Laurent polynomial ring. We make $\kappa\left\{\mathbf{Y}^{\boldsymbol{\theta}}\right\}$ into a $\mathbb{Z}$-graded ring by giving the $i$-th variable $Y_{i}$ degree $\theta_{i}^{\natural}$, and denote the corresponding grading by $\operatorname{deg}_{\boldsymbol{\theta}}(\cdot)$.

Our second convention is the use of a formal variable $\xi$, fixed once and for all. Given a power series $F(\mathbf{x}, \xi) \in \kappa[\mathbf{x}][[\xi]]$ with coefficients in a polynomial ring $\kappa[\mathbf{x}]$ (with $\mathbf{x}$ a possibly countable tuple of variables), we interpret the (formal) equation $F \equiv 0$ as the condition on the $\mathbf{x}$-variables that $F$ be identical zero as a power series in $\xi$. In other words, if $F(\mathbf{x}, \xi)=f_{0}(\mathbf{x})+f_{1}(\mathbf{x}) \xi+$ $f_{2}(\mathbf{x}) \xi^{2}+\ldots$, then $F \equiv 0$ stands for the (infinite) conjunction $f_{0}=f_{1}=$ $f_{2}=\cdots=0$. As $F \equiv 0$ and $\xi^{i} F \equiv 0$ yield equivalent systems of equations, we may extend this to include Laurent polynomials. Similarly, for each $n$, the equivalence $F(\mathbf{x}, \xi) \equiv 0 \bmod \xi^{n}$ stands for the conjunction $f_{0}=f_{1}=\cdots=$ 
$f_{n-1}=0$. An example of a combination of both conventions is

$$
0 \equiv\left(x+y_{\sharp} \xi\right)^{2}+\left(z_{\sharp}+w \xi\right)^{3},
$$

which is equivalent to the conditions

$$
x^{2}+z^{3}=2 x y+3 z^{2} w=y^{2}+3 z w^{2}=w^{3}=0 \quad \text { in } \quad \kappa\left[x, y, \frac{1}{y}, z, \frac{1}{z}, w\right] .
$$

To any variable $X$, we associate its jet variables $\mathbf{x}=\left(x_{0}, x_{1}, \ldots\right)$, denoted by the corresponding lower case letters. Moreover, we identify $X$ with $x_{0}$ (this will correspond to the section of $\rho_{W, n}$ discussed before Theorem 2.1). For $t \in \mathbb{N}_{\sharp}$, we let $\mathbf{x}(t)$ be the twisted tuple of variables $x_{i}$ with $i \geqslant t^{\natural}$. We define the generic jet series of $X$ as

$$
\widehat{x}=X+x_{1} \xi+x_{2} \xi^{2}+\ldots
$$

and its tagged version

$$
\widehat{x}_{\sharp}=X_{\sharp}+x_{1} \xi+x_{2} \xi^{2}+\ldots
$$

Note that only the constant term is actually tagged, which accords with the fact that a power series is invertible if and only if its constant term is. Given $t \in \mathbb{N}_{\sharp}$, we will use the following notational convention: by $\xi^{t} \widehat{x}$, we mean the series $\xi^{t} \widehat{x}$, if $t$ is untagged, and $\xi^{t^{\natural}} \widehat{x}_{\sharp}$, if $t$ is tagged. We extend this practice to tuples of variables $\mathbf{Y}$ component-wise, with corresponding jet variables $\mathbf{y}=\left(\mathbf{Y}, \mathbf{y}_{1}, \mathbf{y}_{2}, \ldots\right)$. In particular, $\mathbf{y}(\boldsymbol{\theta})$ denotes the tuple of all the variables $x_{i}$ with $i \geqslant t^{\natural}$ and $\xi^{\boldsymbol{\theta}} \widehat{\mathbf{y}}$ is the tuple of all $\xi^{t} \widehat{x}$, for $X$ a variable in $\mathbf{Y}$ and $t$ its $\boldsymbol{\theta}$-weight. We put

$$
\kappa\left\{\mathbf{y}^{\boldsymbol{\theta}}\right\}:=\kappa\left\{\mathbf{Y}^{\boldsymbol{\theta}}\right\} \otimes_{\kappa[\mathbf{Y}]} \kappa[\mathbf{y}] .
$$

With these conventions, we can now write down the equations of a jet scheme more succinctly. If $W \subseteq \mathbb{A}_{\kappa}^{m}$ is the closed subscheme defined by the equations $G_{1}(\mathbf{Y})=\cdots=G_{s}(\mathbf{Y})=0$, then $\nabla_{n} W$ is defined by the conditions

$$
G_{1}(\widehat{\mathbf{y}}) \equiv G_{2}(\widehat{\mathbf{y}}) \equiv \cdots \equiv G_{s}(\widehat{\mathbf{y}}) \equiv 0 \quad \bmod \xi^{n} \quad \text { and } \quad \mathbf{y}(\mathbf{n})=0,
$$

where $\mathbf{n} \in \mathbb{N}^{m}$ is the tuple all of whose entries are equal to $n$. Recall that the latter condition simply means that all jet variables $x_{i}=0$ for all $i \geqslant n$ and all variables $X$ in $\mathbf{Y}$. 


\section{Directed jets}

We extend the notion of jet scheme, by considering certain (initial) linear subspaces of jet schemes. Given $\boldsymbol{\theta} \in \mathbb{N}_{\sharp}^{m}$, we define the $n$-th directed jet scheme $\nabla_{n}^{\boldsymbol{\theta}} W$ along $\boldsymbol{\theta}$, as the locally closed subscheme of $\nabla_{n} W$ defined by the conditions $x_{i}=0$ for $i<d^{\natural}$, and $x_{d^{\natural}}$ is invertible if $d$ is tagged, for all variables $X$ in $\mathbf{Y}$ and where $d$ is the weight of $X$ in $\boldsymbol{\theta}$. We may also refer to $\nabla_{n}^{\boldsymbol{\theta}} W$ as the subscheme of all jets along, or with initial direction $\boldsymbol{\theta}$.

Proposition 3.1. If $W \subseteq \mathbb{A}_{\kappa}^{m}$ is the closed subscheme defined by the equations $G_{1}(\mathbf{Y})=\cdots=G_{s}(\mathbf{Y})=0$, and $\boldsymbol{\theta} \in \mathbb{N}_{\sharp}^{m}$, then $\nabla_{n}^{\boldsymbol{\theta}} W$ is isomorphic to the locally closed subscheme given by

$$
G_{1}\left(\xi^{\boldsymbol{\theta}} \hat{\mathbf{y}}\right) \equiv \cdots \equiv G_{s}\left(\xi^{\boldsymbol{\theta}} \hat{\mathbf{y}}\right) \equiv 0 \quad \bmod \xi^{n} \quad \text { and } \quad \mathbf{y}(\mathbf{n}-\boldsymbol{\theta})=0
$$

in $\kappa\left\{\mathbf{y}^{\boldsymbol{\theta}}\right\}[[\xi]]$.

Proof. For each variable $X$ in $\mathbf{Y}$ and $d \in N_{\sharp}^{n}$, let

$$
\widehat{x}(d):=x_{d} \xi^{d^{\natural}}+x_{d^{\natural}+1} \xi^{d^{\natural}+1}+\ldots
$$

where $x_{d}$ is tagged if $d$ is. Let $\widehat{\mathbf{y}}(\boldsymbol{\theta})$ be the tuple of all truncated generic jets $\widehat{x}(d)$ with $d$ the $\boldsymbol{\theta}$-weight of $X$ in $\mathbf{Y}$. Hence, with this notation, the defining equations of $\nabla_{n}^{\boldsymbol{\theta}} W$ are

$$
G_{1}(\widehat{\mathbf{y}}(\boldsymbol{\theta})) \equiv \cdots \equiv G_{s}(\widehat{\mathbf{y}}(\boldsymbol{\theta})) \equiv 0 \bmod \xi^{n}
$$

and $x_{i}=0$ for $i<d$ or $i \geqslant n$. Consider the change of variables $\tau_{\boldsymbol{\theta}}$ given by $x_{i} \mapsto x_{i-d}$, for all $i \geqslant d$ (preserving any tagging), so that $\xi^{\boldsymbol{\theta}} \widehat{\mathbf{y}}=\tau_{\boldsymbol{\theta}}(\widehat{\mathbf{y}}(\boldsymbol{\theta}))$. Applying $\tau_{\boldsymbol{\theta}}$, we get isomorphic equations

$$
\tau_{\boldsymbol{\theta}}\left(G_{1}(\widehat{\mathbf{y}}(\boldsymbol{\theta}))\right) \equiv \cdots \equiv \tau_{\boldsymbol{\theta}}\left(G_{s}(\widehat{\mathbf{y}}(\boldsymbol{\theta}))\right) \equiv 0 \quad \bmod \xi^{n} \quad \text { and } \quad \mathbf{y}(\mathbf{n}-\boldsymbol{\theta})=0,
$$

from which the claim now easily follows.

The second set of equations in (3.2), namely that all $x_{i}=0$ for $i \geqslant n-d^{\natural}$, are called the initial conditions, whereas the first conditions will be called the formal jet equations. 
EXAMPLE 3.2. Recall that $\rho_{W, n}: \nabla_{n} W \rightarrow W$ is the canonical projection of the jet scheme onto the base scheme. Let us calculate the fiber $\rho_{W, n}^{-1}(O)$ of the origin. If $G_{1}=\cdots=G_{s}=0$ are the equations defining $W$, then $\nabla_{n} W$ is given by the equations $G_{i}(\widehat{\mathbf{y}}) \equiv 0 \bmod \xi^{n}$, and $\rho_{W, n}^{-1}(O)$ is the closed subscheme given by $\mathbf{Y}=0$ (recall that $\mathbf{Y}=\mathbf{y}_{0}$ ), that is to say,

$$
\rho_{W, n}^{-1}(O)=\nabla_{n}^{\mathbf{1}} W .
$$

Definition 3.3. Given $\boldsymbol{\theta} \in \mathbb{N}_{\sharp}^{m}$, define the $\boldsymbol{\theta}$-twisted motivic Igusa-zeta series of $W$ to be

$$
\operatorname{Igu}_{W}^{\boldsymbol{\theta}}(t):=\sum_{n=0}^{\infty}\left[\nabla_{n}^{\boldsymbol{\theta}} W\right] t^{n} .
$$

Hence, $\operatorname{Igu}_{W}$ is just the case in which the twist is zero.

As we shall see, for inductive arguments to go through, we will show that all twisted motivic Igusa-zeta series are rational.

\section{Frobenius transforms}

Assume that $\kappa$ has characteristic $p$. Given a closed subscheme $W \subseteq \mathbb{A}_{\kappa}^{m}$ with defining equations $G_{1}=\cdots=G_{s}=0$, its $q$-th Frobenius transform $W^{(q)}$, is the closed subscheme with equations $G_{1}^{q}=\cdots=G_{s}^{q}=0$, where $q$ is some positive power of $p$. One easily verifies that $W^{(q)}$ is well-defined, that is to say, independent from the choice of defining equations. The next result calculates the directed jet scheme of a Frobenius transform:

Lemma 3.4. Given a closed subscheme $W \subseteq \mathbb{A}_{\kappa}^{m}$ over a field $\kappa$ of characteristic $p>0$, a power $q$ of $p$, and a tuple $\boldsymbol{\theta} \in \mathbb{N}_{\sharp}^{m}$, we have an equality

$$
\left[\nabla_{n}^{\boldsymbol{\theta}} W^{(q)}\right]=\left[\nabla_{\left\lceil\frac{n}{q}\right\rceil}^{\boldsymbol{\theta}} W\right] \cdot \mathbb{L}^{m\left(n-\left\lceil\frac{n}{q}\right\rceil\right)}
$$

in $\mathfrak{G} \mathfrak{r}\left(\operatorname{Var}_{\kappa}\right)$, for all $n$.

Proof. Let $G_{1}(\mathbf{Y})=\cdots=G_{s}(\mathbf{Y})=0$ be the equations of $W$, so that $G_{1}^{q}=\cdots=G_{s}^{q}=0$ are the equations of $W^{(q)}$. By $(3.2)$, the defining jet equations of $\nabla_{n}^{\boldsymbol{\theta}} W^{(q)}$ are

$$
G_{1}^{q}\left(\xi^{\boldsymbol{\theta}} \widehat{\mathbf{y}}\right) \equiv \cdots \equiv G_{s}^{q}\left(\xi^{\boldsymbol{\theta}} \widehat{\mathbf{y}}\right) \equiv 0 \bmod \xi^{n},
$$


whereas the initial conditions are $x_{i}=0$ for $i \geqslant n-\operatorname{deg}_{\boldsymbol{\theta}}(X)$ and all variables $X$ in $\mathbf{Y}$. Expanding each $G_{k}\left(\xi^{\boldsymbol{\theta}} \widehat{\mathbf{y}}\right)=\sum_{l} g_{k l}(\mathbf{y}) \xi^{l}$, the jet equations become

$$
\sum_{l} g_{k l}(\mathbf{y})^{q} \xi^{q l} \equiv 0 \quad \bmod \xi^{n} \quad k=1, \ldots, s .
$$

With $a:=\left\lceil\frac{n}{q}\right\rceil$, these equations are equivalent with the condition that all $g_{k l}^{q}(\mathbf{y})=0$ for $l<a$ and $k=1, \ldots, s$. Note that these equations do not involve any of the variables from the initial conditions, that is to say, the $\mathbf{y}(\mathbf{n}-\boldsymbol{\theta})$. Therefore, in $\mathfrak{G} \mathfrak{r}\left(\operatorname{Var}_{\kappa}\right)$, since we may take radicals, their class is the same as the class of the equations $g_{k l}(\mathbf{y})=0$ for $l<a$ and the same initial conditions. On the other hand, by the same argument, the former are equivalent to the jet equations $G_{1}\left(\xi^{\boldsymbol{\theta}} \widehat{\mathbf{y}}\right) \equiv \cdots \equiv G_{s}\left(\xi^{\boldsymbol{\theta}} \widehat{\mathbf{y}}\right) \equiv 0 \bmod \xi^{a}$, that is to say, the jet equations of $\nabla_{a}^{\boldsymbol{\theta}} W$. So remains to count the number of free variables that remain after imposing the initial conditions $\mathbf{y}(\mathbf{a}-\boldsymbol{\theta})=0$ for the $a$-th jet, namely $m(n-a)$, whence the assertion.

Corollary 3.5. Given a closed subscheme $W \subseteq \mathbb{A}_{\kappa}^{m}$ over a field $\kappa$ of characteristic $p>0$, a power $q$ of $p$, and a tuple $\boldsymbol{\theta} \in \mathbb{N}_{\sharp}^{m}$, we have a functional equation among power series

$$
\operatorname{Igu}_{W(q)}^{\theta}(t)=\operatorname{Igu}_{W}^{\theta}\left(t^{q} \mathbb{L}^{m(q-1)}\right) \cdot\left(\sum_{i=0}^{q-1} t^{-i} \mathbb{L}^{-i m}\right)
$$

over $\mathfrak{G}:=\mathfrak{G} \mathfrak{r}\left(\operatorname{Var}_{\kappa}\right)_{\mathbb{L}}$. In particular, $W$ has a rational motivic Igusa-zeta series if and only if some (respectively, any) Frobenius transform does.

Proof. Splitting the sum over all $n \geqslant 1$ by their residue modulo $q$, we get

$$
\begin{aligned}
\operatorname{Igu}_{W^{(q)}}^{\boldsymbol{\theta}}(t) & =\sum_{i=0}^{q-1} \sum_{n \geqslant 1}\left[\nabla_{q n-i}^{\boldsymbol{\theta}} W^{(q)}\right] t^{q n-i} \\
& =\sum_{i=0}^{q-1} \sum_{n \geqslant 1}\left[\nabla_{n}^{\boldsymbol{\theta} W}\right] t^{q n-i} \mathbb{L}^{m(n q-i-n)},
\end{aligned}
$$

where the second equality comes from Lemma 3.4. Factoring out terms not involving $n$ from the inner sum, the assertion follows readily. 


\section{Motivic rationality over a vertex singularity}

By a cone $C$, we mean a homogeneous hypersurface in $\mathbb{A}^{n}$. We call $C$ smooth, if the origin is its only singularity, that is to say, if $\operatorname{Proj}(A)$ is smooth, where $A=\Gamma\left(\mathcal{O}_{C}, C\right)$ is the (graded) affine coordinate ring of $C$. Conversely, any cone is obtained by choosing a polarization, that is to say, an ample line bundle $L$, on a projective hypersurface $W$ and letting $A$ be the section ring $\oplus_{n} H^{0}\left(W, L^{n}\right)$; the cone is smooth if $W$ is. The degree of $C$ is the degree of its defining homogeneous equation, which is equal to the multiplicity at the origin. By a formal (smooth) cone we mean the formal completion $\widehat{C}_{O}$ of a (smooth) cone at the origin. In particular, its ring of global sections is equal to the completion $\widehat{\mathcal{O}}_{C, O}$.

By a vertex singularity, we mean a germ that is analytically isomorphic to a smooth cone, in other words, $(W, P)$ is a vertex singularity if $\widehat{\mathcal{O}}_{W, P} \cong \widehat{\mathcal{O}}_{C, O}$ for some smooth cone $C$. The multiplicity of the vertex is the multiplicity of the origin at the corresponding formal cone. An example of a vertex singularity (of multiplicity two) is an ordinary double point on a planar curve, e.g., $X^{2}+$ $X^{3}+Y^{2}$.

Lemma 4.1. Given a d-dimensional (formal) cone $W$ of degree $e$, the fiber $\rho_{W, n}^{-1}(O)$ above the origin is isomorphic to $\nabla_{n-e} W \times \mathbb{A}^{(d+1)(e-1)}$, for any $n>e$.

Proof. The proof for a formal cone is identical to the affine case, and so we leave the details of the former to the reader. Let $H=0$ be the homogeneous equation of $W$, in the $d+1$ variables $\mathbf{Y}$. By (3.3), the fiber $\rho_{W, n}^{-1}(O)$ is isomorphic to the directed jet scheme $\nabla_{n}^{1} W$, and hence by 3.2 given by

$$
\xi^{e} H(\widehat{\mathbf{y}}) \equiv 0 \quad \bmod \xi^{n} \quad \text { and } \quad \mathbf{y}(\mathbf{n}-\mathbf{1})=0,
$$

for $n>e$. Factoring out $\xi^{e}$ yields the jet equation $H(\widehat{\mathbf{y}}) \equiv 0 \bmod \xi^{n-e}$ of $\nabla_{n-e} W$, and the initial condition has $(d+1)(e-1)$ free variables.

REMARK 4.1. The argument still applies if instead of a single homogeneous equation of degree $e$, we have several such equations, all of the same degree. Put differently, we may take $W$ in the above to be an intersection of (formal) cones of a fixed degree, where $d+1$ is then the embedding dimension of $W$.

TheOREM 4.2. For a d-dimensional scheme $W$ (in arbitrary characteristic) with a (unique) vertex singularity $P$ of degree $e$, its motivic Igusa-zeta series $\operatorname{Igu}_{W}$ over the localization $\mathfrak{G}$ of the Grothendieck ring is rational. More 
precisely, there exists a polynomial $Q$ with coefficients in $\mathfrak{G}$, such that

$$
\operatorname{Igu}_{W}=\frac{Q(t)}{\left(1-\mathbb{L}^{d} t\right)\left(1-\mathbb{L}^{(d+1)(e-1)} t^{e}\right)} .
$$

Proof. By Corollary 2.4 and the definition of vertex singularity, we may reduce to the case that $W$ is a smooth cone. By Corollary 2.2, we have an equality

$$
\left[\nabla_{n} W\right]=[W-P] \cdot \mathbb{L}^{d(n-1)}+\left[\rho_{W, n}^{-1}(P)\right] .
$$

By Lemma 4.1, the last term is equal to $\left[\nabla_{n-m} W\right] \cdot \mathbb{L}^{r}$, where $r:=(d+1)(e-1)$. Multiplying with $t^{n}$, and summing over all $n>e$, we get

$$
\operatorname{Igu}_{W}=p+\frac{[W-P] \cdot \mathbb{L}^{-d}}{\left(1-\mathbb{L}^{d} t\right)}+\mathbb{L}^{r} t^{e} \operatorname{Igu}_{W}
$$

for some polynomial $p$ over $\mathfrak{G}$, accounting for terms with $n \leqslant e$. Solving for $\operatorname{Igu}_{W}$, then shows that the latter is rational with denominator equal to $\left(1-\mathbb{L}^{d} t\right)\left(1-\mathbb{L}^{r} t^{e}\right)$.

\section{The linear rationalization algorithm}

In this section, we fix a hypersurface $W \subseteq \mathbb{A}_{\kappa}^{m}$ defined by a single equation $F(\mathbf{Y})$. If $F$ is not homogeneous, we can no longer expect such a simple relation as in Lemma 4.1 between the jet scheme and the fiber above the singular locus. Write $F$ as a sum of distinct, non-zero monomials $F=\sum_{i} \mu_{i}$.

\section{Twisted initial forms}

As we will shortly see, the following hypersurfaces derived from $W$ will play an important role: for every $\boldsymbol{\theta} \in \mathbb{N}_{\sharp}^{m}$, let $\tilde{W}^{\boldsymbol{\theta}}$ be defined as follows. Let $\operatorname{ord}_{\boldsymbol{\theta}}(F)$, or $\operatorname{ord}_{\boldsymbol{\theta}}(W)$, be the order of $F$ in the grading of $\kappa\left\{\mathbf{Y}^{\boldsymbol{\theta}}\right\}$, that is to say, the minimum of all $\operatorname{deg}_{\boldsymbol{\theta}}\left(\mu_{i}\right)$, and let $\tilde{W}^{\boldsymbol{\theta}}$ be the hypersurface with defining equation

$$
\tilde{F}^{\boldsymbol{\theta}}:=\sum_{\operatorname{deg}_{\boldsymbol{\theta}}\left(\mu_{i}\right)=\operatorname{ord}_{\boldsymbol{\theta}}(W)} \mu_{i}
$$


In particular, $W=\tilde{W}^{\mathbf{0}}$. We call $\tilde{W}^{\boldsymbol{\theta}}$, or rather, $\tilde{F}^{\boldsymbol{\theta}}$, the $\boldsymbol{\theta}$-twisted initial form of $W$. Here is an example to view the previous conventions and definitions at work:

Example 5.1. Let $F=X^{9}+X^{2} Y^{4}+Z^{4}$ and $\boldsymbol{\theta}=\left(2,3^{\sharp}, 5\right)$. Hence $\nabla_{n}^{\left(2,3^{\sharp}, 5\right)} W$ is the locally closed subscheme of $\nabla_{n} W$ given by the conditions $X=x_{1}=Y=y_{1}=y_{2}=Z=z_{1}=z_{2}=z_{3}=z_{4}=0$ and $y_{3}$ is invertible. Using (3.2), its formal jet equations are

$$
\xi^{18} \widehat{x}^{9}+\xi^{16} \widehat{x}^{2} \widehat{y}_{\sharp}^{4}+\xi^{20} \bar{z}^{4} \equiv 0 \bmod \xi^{n}
$$

and $\mathbf{x}(n-2)=\mathbf{y}(n-3)=\mathbf{z}(n-5)=0$. Hence, $\operatorname{ord}_{\left(2,3^{\sharp}, 5\right)}(W)=16$ and the twisted initial form $\tilde{W}^{\left(2,3^{\sharp}, 5\right)}$ is given by $\tilde{F}^{\left(2,3^{\sharp}, 5\right)}=X^{2} Y_{\sharp}^{4}$, that is to say, by the two conditions $X^{2}=0$ and $Y$ is a unit.

\section{Regular base}

We will deduce rationality by splitting off regular pieces of various twisted initial forms, until we arrive at a recursive relation involving the jet scheme of the original hypersurface. We will also consider open subschemes of hypersurfaces, and we refer to these as locally closed hypersurfaces. Given a locally closed hypersurface $W \subseteq \mathbb{A}^{m}$, we say that $\boldsymbol{\theta} \in \mathbb{N}_{\sharp}^{m}$ is $W$-regular if $\tilde{W}^{\boldsymbol{\theta}}$ is regular. For our purposes, it is important to include in this the case that $W$ is given by a monomial in which all variables are tagged, since this defines the empty scheme, which we consider to be regular! As with jets, directed jets above regular base have a locally trivial fibration, a fact which will allow us to determine their contribution to the Igusa-zeta series.

Proposition 5.2. Let $W \subseteq \mathbb{A}^{m}$ be a locally closed hypersurface. For each $W$-regular tuple $\boldsymbol{\theta} \in \mathbb{N}_{\sharp}^{m}$, we have an equality

$$
\left[\nabla_{n}^{\boldsymbol{\theta}} W\right]=\left[\tilde{W}^{\boldsymbol{\theta}}\right] \cdot \mathbb{L}^{(m-1)(n-1)+\operatorname{ord}_{\boldsymbol{\theta}}(W)-|\boldsymbol{\theta}|}
$$

in $\mathfrak{G r}\left(\operatorname{Var}_{\kappa}\right)$.

Proof. Let $F$ be the defining equation of $W$ and write it as a sum of distinct, non-zero monomials $F=\sum_{i} \mu_{i}$. Let us put $a:=\operatorname{ord}_{\boldsymbol{\theta}}(W)$; recall that it is the minimum of all $\operatorname{deg}_{\boldsymbol{\theta}}\left(\mu_{i}\right)$. Let $G:=\tilde{F}^{\boldsymbol{\theta}}$ and $H:=F-G$. By 3.2 , the formal jet equation of $\nabla_{n}^{\boldsymbol{\theta}} W$ is

$$
\xi^{a} G(\widehat{\mathbf{y}})+\xi^{a+1} H(\widehat{\mathbf{y}}) \equiv 0 \quad \bmod \xi^{n}
$$


whereas the initial condition is $\mathbf{y}(\mathbf{n}-\boldsymbol{\theta})=0$. Leaving aside for now the latter initial conditions, factoring out $\xi^{a}$, yields the formal jet equation

$$
G(\widehat{\mathbf{y}})+\xi H(\widehat{\mathbf{y}}) \equiv 0 \bmod \xi^{n-a} .
$$

Compare this with the formal jet equation of $\nabla_{n-a} \tilde{W}^{\boldsymbol{\theta}}$ given by

$$
G(\widehat{\mathbf{y}}) \equiv 0 \quad \bmod \xi^{n-a} .
$$

Let $g_{l}$ and $h_{l}$ be the (untwisted) jet equations of $G$ and $H$ respectively, that is to say, $G(\widehat{\mathbf{y}})=\sum g_{l}(\mathbf{y}) \xi^{l}$ and $H(\widehat{\mathbf{y}})=\sum h_{l}(\mathbf{y}) \xi^{l}$. Using (5.1), it is now easy to see that the jet equations of $\nabla_{n}^{\boldsymbol{\theta}} W$ are

$$
g_{l}(\mathbf{y})+h_{l-1}(\mathbf{y})=0
$$

for $l=0, \ldots, n-a-1$, whereas those for $\nabla_{n-a} \tilde{W}^{\boldsymbol{\theta}}$ are

$$
g_{l}(\mathbf{y})=0
$$

for $l=0, \ldots, n-a-1$. Recall that $G=g_{0}, H=h_{0}$, and each $g_{l}, h_{l}$ only depends on the tuples of jet variables $\mathbf{y}_{0}=\mathbf{Y}, \mathbf{y}_{1}, \ldots, \mathbf{y}_{l-1}$. In [18, Theorem 4.14], we showed that the $g_{l}$ with $l>0$ are linear in the $\mathbf{y}_{l-1}$, and we can locally solve for one of these variables. Let us reprove this result by showing that, for each $l>0$, we have

$$
g_{l}(\mathbf{y})=\sum_{X \text { in } \mathbf{Y}} x_{l-1} \frac{\partial G}{\partial X}+q_{l}
$$

where $q_{l}$ is a polynomial depending only on the $\mathbf{y}_{i}$ with $i<l-1$. Indeed, write $\widehat{\mathbf{y}}=\breve{\mathbf{y}}+\xi^{l-1} \mathbf{y}_{l-1}$, where $\breve{\mathbf{y}}$ is the truncated generic jet given as $\sum_{i<l-1} \xi^{i} \mathbf{y}_{i}$. By Taylor expansion, we get

$$
G(\widehat{\mathbf{y}}) \equiv G(\breve{\mathbf{y}})+\left.\sum_{X \text { in } \mathbf{Y}} x_{l-1} \xi^{l-1} \frac{\partial G}{\partial X}\right|_{\breve{\mathbf{y}}} \bmod \xi^{l} .
$$

Since $\mathbf{Y} \equiv \breve{\mathbf{y}} \bmod \xi$, the formal jet equation therefore becomes

$$
G(\breve{\mathbf{y}})+\sum x_{l-1} \xi^{l-1} \frac{\partial G}{\partial X} \equiv 0 \bmod \xi^{l} .
$$

In the expansion of $G(\breve{\mathbf{y}})$ into powers of $\xi$, the coefficient in front of $\xi^{l-1}$ only depends on the $\mathbf{y}_{i}$ for $i<l-1$, whence our claim (5.5).

Now, since we assumed $G$ to be regular, the basic open sets $U_{X}$ given by inverting $\partial G / \partial X$ for each variable $X$ in $\mathbf{Y}$ form a covering of the hypersurface 
determined by $G$. On $U_{X}$, using (5.5), we can solve for the jet variable $x_{l-1}$ in (5.4), and for the same reason, also in (5.3), since $h_{l-1}$ only depends on the jet variables $\mathbf{y}_{i}$ with $i<l-1$. In other words, $\nabla_{n}^{\boldsymbol{\theta}} W$ and $\nabla_{n-a} \tilde{W}^{\boldsymbol{\theta}}$ have isomorphic jet equations on each basic open $U_{X}$. On the other hand, their respective initial conditions are $\mathbf{y}(\mathbf{n}-\boldsymbol{\theta})=0$ and $\mathbf{y}(\mathbf{n}-\mathbf{a})=0$, which is a difference of $|\mathbf{a}-\boldsymbol{\theta}|=m a-|\theta|$ conditions. Moreover, our argument also shows, as in [18, Theorem 4.14], that $\nabla_{n-a} \tilde{W}^{\boldsymbol{\theta}}$ and $\tilde{W}^{\boldsymbol{\theta}} \times \mathbb{A}^{(n-a-1)(m-1)}$ are locally isomorphic, on each basic open $U_{X}$. The desired equality in the Grothendieck ring follows now easily from these local calculations (see for instance [18, Lemma 4.13]).

\section{Recursion}

Given $\boldsymbol{\alpha}, \boldsymbol{\beta} \in \mathbb{N}_{\sharp}^{m}$, we will write $\boldsymbol{\alpha} \triangleleft_{W} \boldsymbol{\beta}$, if $\boldsymbol{\alpha} \leq \boldsymbol{\beta}$ and there exists some $s>0$ such that

$$
F\left(\xi^{\boldsymbol{\beta}} \hat{\mathbf{y}}\right)=\xi^{s} F\left(\xi^{\boldsymbol{\alpha}} \widehat{\mathbf{y}}\right)
$$

An easy calculation shows that necessarily $s=\operatorname{ord}_{\boldsymbol{\beta}}(W)-\operatorname{ord}_{\boldsymbol{\alpha}}(W)$. The definition also implies that $\boldsymbol{\alpha}$ and $\boldsymbol{\beta}$ have the same tagged entries. Note that $F$ is homogeneous in the classical sense if and only if $\mathbf{0} \triangleleft_{W} \mathbf{1}$.

Lemma 5.3. If $\boldsymbol{\alpha} \triangleleft_{W} \boldsymbol{\beta}$, then

$$
\left[\nabla_{n}^{\boldsymbol{\beta}} W\right]=\left[\nabla_{n-s}^{\boldsymbol{\alpha}} W\right] \cdot \mathbb{L}^{s m-|\boldsymbol{\beta}|+|\boldsymbol{\alpha}|}
$$

in $\mathfrak{G}$, for all $n>s$, with $s=\operatorname{ord}_{\boldsymbol{\beta}}(W)-\operatorname{ord}_{\boldsymbol{\alpha}}(W)$.

Proof. By 3.2 , the formal jet equations of $\nabla_{n}^{\boldsymbol{\beta}} W$ are

$$
F\left(\xi^{\boldsymbol{\beta}} \hat{\mathbf{y}}\right) \equiv 0 \quad \bmod \xi^{n} \quad \text { and } \quad \mathbf{y}(\mathbf{n}-\boldsymbol{\beta})=0 .
$$

By assumption, the power series in the formal jet equation equals $\xi^{s} F\left(\xi^{\alpha} \widehat{\mathbf{y}}\right)$, and so yields the formal jet equation

$$
F\left(\xi^{\alpha} \widehat{\mathbf{y}}\right) \equiv 0 \quad \bmod \xi^{n-s} .
$$

However, (5.7) is also the jet equation of $\nabla_{n-s}^{\alpha} W$, again by (3.2). As the initial condition for $\nabla_{n-s}^{\boldsymbol{\alpha}} W$ is given by $\mathbf{y}(\mathbf{n}-\mathbf{s}-\boldsymbol{\alpha})=0$, the difference between the two directed jet schemes lies in the number of free variables not covered by the respective initial conditions, a number equal to $|\mathbf{s}-\boldsymbol{\beta}+\boldsymbol{\alpha}|=s m-|\boldsymbol{\beta}|+|\boldsymbol{\alpha}|$, whence the assertion. 


\section{Rationalizing trees}

We are interested in subtrees of $\mathbb{N}_{\sharp}^{m}$, and will use the following terminology: by a tree we mean a connected partially ordered subset of (nodes from) $\mathbb{N}_{\sharp}^{m}$ such that any initial segment is totally ordered. The unique minimum is called the root of the tree, and any maximal element is called a leaf. By a branch, we will mean a chain from a node $\boldsymbol{\alpha}$ to a leaf $\boldsymbol{\beta}$. By the way we defined the ordering on $\mathbb{N}_{\sharp}^{m}$, if the $i$-th entry of a node is tagged, then any node above it has the same $i$-th entry. In particular, the tagged entries of the root never change.

To describe the various successor functions on the trees we will construct, denote by $e_{i}$, for each $i$, the function on $\mathbb{N}_{\sharp}^{m}$ which increases the $i$-th entry by one (note that per our addition convention, each entry stays in whichever state, tagged or untagged, it was), and by $e_{i}^{\sharp}$, the function which tags the $i$-th entry but leaves the remaining entries unchanged. As we often label by means of variables rather than indices, we write instead $e_{X}$ and $e_{X}^{\sharp}$, if $X$ is the $i$-th variable in an $m$-tuple of variables $\mathbf{Y}$.

Given a binary vector $\varepsilon$, we let $e_{\boldsymbol{\varepsilon}}$ (respectively, $e_{\boldsymbol{\varepsilon}}^{\sharp}$ ) be the composition of all $e_{i}$ (respectively, all $e_{i}^{\sharp}$ ) for which $\varepsilon_{i}=1$. Note that all these transformations commute with each other. Finally, for two binary vectors $\boldsymbol{\delta} \leq \boldsymbol{\eta}$, we let $e_{\boldsymbol{\delta}}^{\boldsymbol{\eta}}$ be the composition of $e_{\boldsymbol{\delta}}$ and $e_{\boldsymbol{\eta}-\boldsymbol{\delta}}^{\sharp}$. For instance,

$$
\begin{aligned}
e_{(0,0,0,1,0)}^{(1,1,0,1,0)}\left(2,3^{\sharp}, 1,4,1\right)=e_{(1,1,0,0,0)}^{\sharp} & e_{(0,0,0,1,0)}\left(2,3^{\sharp}, 1,4,1\right) \\
& =e_{1}^{\sharp} e_{2}^{\sharp} e_{4}\left(2,3^{\sharp}, 1,4,1\right)=\left(2^{\sharp}, 3^{\sharp}, 1,5,1\right) .
\end{aligned}
$$

Note that $e_{\boldsymbol{\delta}}^{\boldsymbol{\eta}}(\boldsymbol{\theta})$ has underlying value equal to $\boldsymbol{\theta}^{\natural}+\boldsymbol{\delta}$. More precisely, taking in account our addition convention, we have

$$
e_{\boldsymbol{\delta}}^{\boldsymbol{\eta}}(\boldsymbol{\theta})=e_{\boldsymbol{\eta}-\boldsymbol{\delta}}^{\sharp}(\boldsymbol{\theta}+\boldsymbol{\delta}) .
$$

Note that $e_{\delta}^{\eta}$ can fail to be an increasing function (if in the above example we replace $(0,0,0,1,0)$ by $(0,1,0,1,0)$ the resulting tuple is $\left(2^{\sharp}, 4^{\sharp}, 1,5,1\right)$, which is not comparable with $\left(2,3^{\sharp}, 1,4,1\right)$ because the second entries are both tagged but distinct). We do have

$$
\left.(\forall i) \text { [if } \theta_{i} \text { tagged then } \eta_{i}=0\right] \Rightarrow \boldsymbol{\theta} \leq e_{\boldsymbol{\delta}}^{\boldsymbol{\eta}}(\boldsymbol{\theta}) .
$$

We will use these transformations mainly through the following result. 
Lemma 5.4. Let $W \subseteq \mathbb{A}_{\kappa}^{m}$ be a closed subscheme. For every $\boldsymbol{\theta} \in \mathbb{N}_{\sharp}^{m}$, and every binary vector $\boldsymbol{\eta}$, we have an identity

$$
\left[\nabla_{n}^{\boldsymbol{\theta}} W\right]=\sum_{\gamma \leq \boldsymbol{\eta}}\left[\nabla_{n}^{e_{\gamma}^{\eta}(\boldsymbol{\theta})} W\right]
$$

in $\mathfrak{G} \mathfrak{r}\left(\operatorname{Var}_{\kappa}\right)$, for all $n$.

Proof. Let $\mathbf{Y}$ be the variables defining $W$. Let us derive first the following equality in $\mathfrak{G r}\left(\operatorname{Var}_{\kappa}\right)$ for a given variety $V$. For binary vectors $\boldsymbol{\gamma} \leq \boldsymbol{\eta}$, let $\bar{V}_{\boldsymbol{\gamma}}^{\boldsymbol{\eta}}$ be the locally closed subvariety obtained from $V$ by adding, for every variable $X$ in $\mathbf{Y}$ such that $\operatorname{deg}_{\boldsymbol{\eta}}(X)=1$, the condition that $X=0$ if $\operatorname{deg}_{\boldsymbol{\gamma}}(X)=0$, and the condition that $X$ is invertible, if $\operatorname{deg}_{\gamma}(X)=1$. As $\gamma$ runs over all binary vectors with $\boldsymbol{\gamma} \leq \boldsymbol{\eta}$, the $\bar{V}_{\boldsymbol{\gamma}}^{\boldsymbol{\eta}}$ form a partition of $V$, and so

$$
[V]=\sum_{\gamma \leq \boldsymbol{\eta}}\left[\bar{V}_{\gamma}^{\eta}\right]
$$

Apply this to $V:=\nabla_{n}^{\theta} W$. Since by $(3.2)$, the formal jet equations for $V$ are $F_{i}\left(\xi^{\boldsymbol{\theta}} \widehat{\mathbf{y}}\right) \equiv 0 \bmod \xi^{n}$, for $i=1, \ldots, s$, where $F_{1}=\cdots=F_{s}=0$ are the defining equations of $W$, the formal jet equations of $\bar{V}_{\boldsymbol{\gamma}}^{\boldsymbol{\eta}}$ are $F_{i}\left(\xi^{\boldsymbol{\theta}+\boldsymbol{\eta}-\boldsymbol{\gamma}} \widehat{\mathbf{y}}\right) \equiv 0$ $\bmod \xi^{n}$, for $i=1, \ldots, s$, together with inverting all $X$ for which $\operatorname{deg}_{\gamma}(X)=1$. As these are precisely the formal jet equations for $\nabla_{n}^{e_{\eta-\gamma}^{\eta}(\boldsymbol{\theta})} W$, we proved the assertion (note that summing over all $\gamma$ is the same as summing over all $\eta-\gamma)$.

We define by induction on the height of a tree in $\mathbb{N}_{\sharp}^{m}$ for it to be a resolution tree as follows: any singleton is a resolution tree; if $\mathcal{T}$ is a resolution tree, then so is $\mathcal{T}^{\prime}$ which is obtained from $\mathcal{T}$ first by choosing a leaf $\gamma$ of $\mathcal{T}$ and a binary vector $\boldsymbol{\eta}$ such that whenever an entry $\gamma_{i}$ is tagged, the corresponding entry $\eta_{i}$ is zero, and then by adding on to $\mathcal{T}$ at $\boldsymbol{\gamma}$ all the $e_{\boldsymbol{\delta}}^{\boldsymbol{\eta}}(\boldsymbol{\gamma})$ as new leafs, for $\boldsymbol{\delta} \leq \boldsymbol{\eta}$. By (5.8), the new subset is indeed a tree. In particular, if every entry of some node $\boldsymbol{\theta} \in \mathcal{T}$ is tagged and $\mathcal{T}$ is a resolution tree, then $\boldsymbol{\theta}$ is necessarily a leaf of $\mathcal{T}$. Moreover, any truncation of a resolution tree $\mathcal{T}$, that is to say, all nodes of $\mathcal{T}$ greater than or equal to a fixed node, is again a resolution tree.

Lemma 5.5. Let $W \subseteq \mathbb{A}^{m}$ be a closed subscheme and let $\mathcal{T} \subseteq \mathbb{N}_{\sharp}^{m}$ be a finite subtree with root $\boldsymbol{\theta}$. If $\mathcal{T}$ is a resolution tree, then

$$
\left[\nabla_{n}^{\boldsymbol{\theta}} W\right]=\sum_{\gamma \in \mathcal{T} \text { leaf }}\left[\nabla_{n}^{\gamma} W\right]
$$


in $\mathfrak{G} \mathfrak{r}\left(\operatorname{Var}_{\kappa}\right)$, for all $n$. In particular, we have an identity among motivic zeta series

$$
\operatorname{Igu}_{W}^{\boldsymbol{\theta}}=\sum_{\gamma \in \mathcal{T}} \operatorname{Igu}_{W}^{\gamma}
$$

Proof. An easy induction on the height of a node, using Lemma 5.4 . yields the first assertion. The second then follows by multiplying with $t^{n}$ and summing over all $n$.

Definition 5.6. We say that a finite tree $\mathcal{T} \subseteq \mathbb{N}_{\sharp}^{m}$ is $W$-rationalizing, for some closed subscheme $W \subseteq \mathbb{A}^{m}$, if for every leaf $\gamma$, either $\gamma$ is $W$-regular or we can find $\boldsymbol{\theta}<\boldsymbol{\gamma}$ in $\mathcal{T}$ such that $\boldsymbol{\theta} \triangleleft_{W} \boldsymbol{\gamma}$.

THEOREM 5.7. If $\mathcal{T}$ is a $W$-rationalizing resolution tree with root $\boldsymbol{\theta} \in \mathbb{N}_{\sharp}^{m}$, then the twisted Igusa-zeta series $\operatorname{Igu}_{W}^{\theta}$ is rational over $\mathfrak{G}:=\mathfrak{G r}\left(\operatorname{Var}_{\kappa}\right)_{\mathbb{L}}$.

Proof. By (5.10), it suffices to show that $\operatorname{Igu}_{W}^{\gamma}$ is rational over $\mathfrak{G}$, for every leaf $\gamma$ of $\mathcal{T}$, and we will do this by induction on the number $u(\gamma)$ of untagged entries. If $u(\gamma)=0$, then $\gamma$ is necessarily $W$-regular, and hence by Proposition 5.2, we have

$$
\left[\nabla_{n}^{\gamma} W\right]=\left[\tilde{W}^{\gamma}\right] \cdot \mathbb{L}^{(m-1) n+r}
$$

in $\mathfrak{G r}\left(\operatorname{Var}_{\kappa}\right)$, where $r:=\operatorname{ord}_{\boldsymbol{\gamma}}(W)-|\gamma|-m+1$. Multiplying with $t^{n}$ and summing then yields

$$
\operatorname{Igu}_{W}^{\gamma}=\frac{\mathbb{L}^{r}\left[\tilde{W}^{\gamma}\right]}{1-\mathbb{L}^{m-1}}
$$

For a general leaf $\gamma$, we are done by the same argument if it is $W$-regular, and so we may assume that $\delta \triangleleft_{W} \gamma$, for some node $\boldsymbol{\delta}$. By definition, $\boldsymbol{\delta}$ has the same tagged entries as $\gamma$. Moreover, by definition of resolution tree, any successor of $\boldsymbol{\delta}$ not on the branch to $\boldsymbol{\gamma}$ has fewer untagged variables. In particular, any other leaf lying above $\boldsymbol{\delta}$ has fewer untagged variables and hence by induction, its motivic Igusa zeta series is rational. On the other hand, by Lemma 5.3, the directed jet class along $\gamma$ is given by

$$
\left[\nabla_{n}^{\gamma} W\right]=\left[\nabla_{n-s}^{\delta} W\right] \cdot \mathbb{L}^{r}
$$

in $\mathfrak{G}$, for all $n>s$, with $s:=\operatorname{ord}_{\boldsymbol{\gamma}}(W)-\operatorname{ord}_{\boldsymbol{\delta}}(W)$ and $r:=s m-|\boldsymbol{\gamma}|+|\boldsymbol{\delta}|$. Multiplying with $t^{n}$ and summing over all $t$, we get an identity

$$
\operatorname{Igu}_{W}^{\gamma}=p+\mathbb{L}^{r} t^{s} \operatorname{Igu}_{W}^{\delta},
$$


where $p$ is some polynomial in $t$ (accounting for the small values of $n$ ). By (5.10), 5.11) and our induction hypothesis applied to the truncation of $\mathcal{T}$ at $\delta$, we therefore have an identity

$$
\operatorname{Igu}_{W}^{\boldsymbol{\delta}}=Q+\operatorname{Igu}_{W}^{\gamma}=Q+p+\mathbb{L}^{r} t^{s} \operatorname{Igu}_{W}^{\boldsymbol{\delta}}
$$

for some rational series $Q$ over $\mathfrak{G}$, stemming from the remaining leafs. Solving for $\operatorname{Igu}_{W}^{\delta}$ then proves its rationality, whence also that of $\operatorname{Igu}_{W}^{\gamma}$ by (5.11), as we needed to show.

\section{Linear singularities}

The algorithm that we will use here to construct an $W$-rationalizing resolution tree with root $\mathbf{0}$, thus establishing the rationality of the motivic Igusa-zeta series of a hypersurface $W$ by Theorem 5.7, relies on the simple form the singular locus takes. Namely, we say that a hypersurface $W$ has linear singularities at a closed point $P$, if, after a translation of $P$ to the origin, the singular locus of $W$ is contained in a finite union of coordinate subspaces, where a coordinate subspace is a closed subscheme given by equations $Y_{i_{1}}=\cdots=Y_{i_{s}}=0$ for some subset $Y_{i_{j}}$ of the variables. To be more precise, define the Milnor ideal $\mathfrak{M i l}_{W}$ of $W$ as the radical of the ideal generated by $F$ and all its partial derivatives, where $F$ is the defining equation of $W$. We will apply the algorithm to hypersurfaces all of whose twisted initial forms have linear singularities; in the literature, this condition is commonly known as being non-degenerated with respect to its Newton polyhedron.

Lemma 5.8. A hypersurface $W$ is regular if and only if its Milnor ideal is the unit ideal. Moreover, $W$ is non-degenerated with respect to its Newton polyhedron if and only if the Milnor ideal of every initial form contains a monomial.

Proof. The first assertion is the well-known Jacobian criterion for smoothness (see, for instance, [14, Theorem 30.3] or [8, Corollary 16.20]). The second assertion is just a rephrasing of the definition.

\section{Single-branch linear rationalization algorithm for diagonal hypersurfaces with an isolated singularity}

In its simplest form, the algorithm works as follows: assume for every twisted initial form $\tilde{W}^{\theta}$ of $W$, there exists a variable $X$ such that the basic subset $\left(\tilde{W}^{\theta}\right)_{X}$ is smooth (depending on the situation, we may have to choose 
such a variable more carefully, as will be the case in $\$ 7$ below). We then apply Lemma 5.4 with $\boldsymbol{\eta}$ the binary vector giving weight one to $X$ and zero to the remaining variables. In its simplest implementation, any tagged node will be a leaf, and, in fact $W$-regular, whence requires no further action. We continue this process (on the remaining untagged leaf) until we reach an untagged leaf $\gamma$ with $\mathbf{0} \triangleleft_{W} \gamma$, at which point we can invoke Theorem 5.7. If such a leaf $\gamma$ can be found, we say that the algorithm stops.

To illustrate our algorithm, let us look at a hypersurface $W$ with an equation of the form

$$
F:=r_{1} Y_{1}^{a_{1}}+\cdots+r_{m} Y_{m}^{a_{m}}
$$

with $a_{i}>0$ and $r_{i} \in \kappa$; we will refer to such a $W$ as a diagonal hypersurface. In characteristic zero, the origin is an isolated singularity, but in positive characteristic, this is only the case if at most one of the powers $a_{i}$ is divisible by the characteristic. In the isolated singularity case, the algorithm as described above does apply: any twisted initial form is again a diagonal hypersurface; if it is one of the powers $Y_{i}^{a_{i}}$, its regular locus, although empty, is obtained by inverting $Y_{i}$, even if $a_{i}$ is divisible by the characteristic; in the remaining case, we can always invert one variable whose power is not divisible by the characteristic, yielding a smooth twisted initial form. So remains to show that this algorithm stops, that is to say, will eventually produce a leaf $\gamma$ such that $\mathbf{0} \triangleleft_{W} \boldsymbol{\gamma}$. To see this, note that the set of all $\operatorname{ord}_{\boldsymbol{\theta}}(W)$, with $\boldsymbol{\theta}$ running over all untagged nodes in the tree, is equal to the union of the semi-groups $a_{i} \mathbb{N}$, for $i=1, \ldots, m$. Therefore, if $e$ is the least common multiple of all $a_{i}$, it will occur as some $\operatorname{ord}_{\boldsymbol{\gamma}}(W)$ for some untagged leaf $\boldsymbol{\gamma}$ in this algorithm. It is now easy to see that $\tilde{W}^{\gamma}=W$, and hence we showed:

TheOREM 5.9. The motivic Igusa zeta-series $\operatorname{Igu}_{W}$ of a diagonal hypersurface $W$ with an isolated singularity is rational over $\mathfrak{G}$.

In the next section, we will work out in complete detail the implementation of this algorithm for the diagonal surface $X^{2}+Y^{3}+Z^{4}=0$. Generalizing these calculations, we will derive the following formula:

Corollary 5.10. If $r_{1} Y_{1}^{a_{1}}+\cdots+r_{m} Y_{m}^{a_{m}}=0$ is the equation of the diagonal hypersurface $W$ with an isolated singularity, then there exists a polynomial $Q_{W}(t) \in \mathfrak{G}[t]$ such that

$$
\operatorname{Igu}_{W}=\frac{Q_{W}(t)}{\left(1-\mathbb{L}^{m-1} t\right)\left(1-\mathbb{L}^{N} t^{e}\right)}
$$


where $e$ is the least common multiple of $a_{1}, \ldots, a_{m}$, and where

$$
N=e\left(\frac{a_{1}-1}{a_{1}}+\cdots+\frac{a_{m}-1}{a_{m}}\right) .
$$

\section{Multi-branch linear rationalization algorithm for monomial hypersurfaces}

Instead of choosing one variable at a time occurring as a monomial in the Milnor ideal, Lemma 5.4 allows us to choose several, or even all, to create a new level in the resolution tree. We will see this at work in more detail in $\$ 6$, but just as an illustration, let us use this variant of the algorithm to calculate the motivic Igusa zeta series of a monomial hypersurface $W$, that is to say, defined by an equation $\mathbf{X}^{\boldsymbol{\nu}}=0$, with $\mathbf{X}$ an $m$-tuple of variables. If the characteristic is $p$, then we can apply Corollary 3.5. to reduce to the case that $\nu$ is not a multiple of $p$. For simplicity, let us assume that no variable occurs linearly, so that the Milnor ideal of $W$ is just the product of all variables. Hence, we will apply Lemma 5.4 with $\boldsymbol{\eta}=\mathbf{1}$, so that $\gamma$ runs over all binary vectors. For $\gamma=\mathbf{1}$, homogeneity yields $\mathbf{0} \triangleleft \mathbf{1}$ so that $\mathbf{1}$ is designated as a leaf by the algorithm, that is to say, we are done with this branch. The remaining $\gamma$ have at least one tagged entry, and we now proceed by induction. For instance, if $W$ is the planar curve defined by $X^{a} Y^{b}=0$, then the algorithm produces the following tree
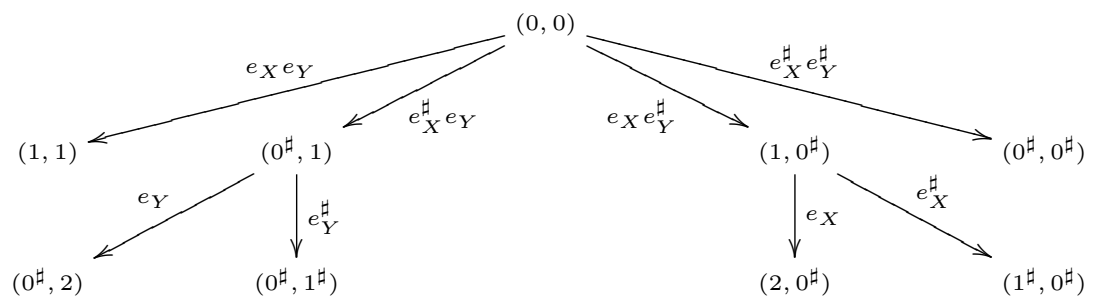

Figure 1. The rationalization tree for the $X^{a} Y^{b}=0$

Since the leafs all of whose entries are tagged have empty initial form, they do not contribute, whereas we have recursion relations $(0,0) \triangleleft(1,1)$, $\left(0^{\sharp}, 1\right) \triangleleft\left(0^{\sharp}, 2\right)$, and $\left(1,0^{\sharp}\right) \triangleleft\left(2,0^{\sharp}\right)$. Apply Lemma 5.5 to the two truncations above the nodes $\left(0^{\sharp}, 1\right)$ and $\left(0,1^{\sharp}\right)$ respectively, and use (5.11), to get

$$
\operatorname{Ig} u_{W}^{\left(0^{\sharp}, 1\right)}=\frac{p_{(0,1)}}{\left(1-t^{b} \mathbb{L}^{2 b-1}\right)} \quad \text { and } \quad \operatorname{Igu}_{W}^{\left(1,0^{\sharp}\right)}=\frac{p_{(1,0)}}{\left(1-t^{a} \mathbb{L}^{2 a-1}\right)},
$$


for some polynomials $p_{(0,1)}$ and $p_{(1,0)}$ over $\mathfrak{G}$. Another application of Lemma 5.5, this time to the entire tree, gives

$$
\operatorname{Igu}_{W}=\frac{p_{(1,0)}}{\left(1-t^{a} \mathbb{L}^{2 a-1}\right)}+\frac{p_{(0,1)}}{\left(1-t^{a} \mathbb{L}^{2 a-1}\right)}+\operatorname{Igu}_{W}^{(1,1)} .
$$

Using (5.11) once more than yields

$$
\operatorname{Igu}_{W}=\frac{p_{(1,1)}+\frac{p_{(1,0)}}{\left(1-t^{a, \mathbb{L}^{2 a-1}}\right)}+\frac{p_{(0,1)}}{\left(1-t^{a, 1} \mathbb{L}^{2 a-1}\right)}}{\left(1-t^{a+b} \mathbb{L}^{2 a+2 b-2}\right)}
$$

for some polynomial $p_{(1,1)}$. It is rather obvious how this generalizes to several variables, and so, without further argument, we state:

TheOREM 5.11. Let $W \subseteq \mathbb{A}^{m}$ be a monomial hypersurface given by $\mathbf{X}^{\boldsymbol{\nu}}=0$ with $\boldsymbol{\nu}=\left(\nu_{1}, \ldots, \nu_{m}\right)$ not a multiple of the characteristic. Then $\operatorname{Igu}_{W}$ is a rational function over $\mathfrak{G}$ with denominator given as the product of all $(1-$ $\left.t^{a} \mathbb{L}^{a m-s}\right)$, where a runs over all s-fold sums $\nu_{i_{1}}+\cdots+\nu_{i_{s}}$, with $1 \leqslant i_{1}<$ $\cdots<i_{s} \leqslant m$ and $s=1, \ldots, m$.

\section{Motivic rationality over Du Val surfaces}

In this section, we implement a multi-branching algorithm to calculate explicitly the motivic Igusa zeta series of Du Val surfaces, which over a field $\kappa$ of characteristic different $p \neq 2$, are precisely the isolated canonical singularities (at the origin $O$ ). Over $\mathbb{C}$, they can be realized, up to analytic isomorphism, as the quotients $\mathbb{A}^{2} / \Gamma$, where $\Gamma \subseteq \mathrm{SL}_{2}(\mathbb{C})$ is a finite subgroup. A complete invariant is the dual resolution graph viewed as one of the following Dynkyn diagrams: $A_{k}, D_{k}, E_{6}, E_{7}$, or $E_{8}$, and we therefore will denote them simply by the latter letters. The main result of this section is the rationality of their motivic Igusa-zeta series over $\mathfrak{G}$, summarized by the following table, where we listed in the last column only the relevant factor in the denominator (the other factor being $\left.\left(1-\mathbb{L}^{2} t\right)\right)$.

If $\kappa$ has characteristic $p=2$, then $A_{k}, D_{k}$ and $E_{6}$ no longer have an isolated singularity, the only condition which is relevant in the proof below. A change of variables, however, always reduces their equation to a surface defined by an (almost separable) poly-diagonal (see \$7), the rationality of which we will establish below. For instance, taking the least obvious case, $D_{k}$ when $k$ is even, make the change of variables $Y \mapsto Y+Z^{\frac{k-2}{2}}$, yielding the almost separable poly-diagonal $X^{2}+Y^{2} Z=0$. Therefore, in the sequel, we will assume $p \neq 2$. 
Table 1. Denominator of the Igusa-zeta series for Du Val surfaces $(p \neq 2)$

\begin{tabular}{|c|r|c|c|}
\hline \multicolumn{2}{|c|}{ Du Val surface $W$} & equation & denominator of $\left(1-\mathbb{L}^{2} t\right) \operatorname{Igu}_{W}$ \\
\hline \hline \multirow{2}{*}{$A_{k}$} & $k$ odd & \multirow{2}{*}{$X^{2}+Y^{2}+Z^{k+1}$} & $\left(1-\mathbb{L}^{2 k+1} t^{k+1}\right)$ \\
\cline { 4 - 5 } & $k$ even & & $\left(1-\mathbb{L}^{4 k+2} t^{2 k+2}\right)$ \\
\hline \multicolumn{2}{|c|}{$D_{k}$} & $X^{2}+Y^{2} Z+Z^{k-1}$ & $\left(1-\mathbb{L}^{4 k-5} t^{2 k-2}\right)$ \\
\hline \multicolumn{2}{|c|}{$E_{6}$} & $X^{2}+Y^{3}+Z^{4}$ & $\left(1-\mathbb{L}^{23} t^{12}\right)$ \\
\hline \multicolumn{2}{|c|}{$E_{7}$} & $X^{2}+Y^{3}+Y Z^{3}$ & $\left(1-\mathbb{L}^{29} t^{18}\right)$ \\
\hline$E_{8}$ & $X^{2}+Y^{3}+Z^{5}$ & $\left(1-\mathbb{L}^{60} t^{30}\right)$ \\
\hline
\end{tabular}

\section{The $E_{6}$-surface}

Let us work step-by-step through the rationalization algorithm for the Du Val surface $E_{6}$ with equation $X^{2}+Y^{3}+Z^{4}$. Being a diagonal surface, its motivic rationality follows from Corollary 5.10 , but we will use a slight modification by allowing multi-branching (that is to say, tagging possibly more than one variable at the same time). We first take a 'short-cut' by observing that the origin $O$ is an isolated singularity, so that we only need to calculate the class of $\nabla_{n}^{(1,1,1)} E_{6}=\rho_{E_{6}, n}^{-1}(O)$ by Corollary 2.2 . By 3.2 , its formal jet equations are

$$
\xi^{2} \widehat{x}^{2}+\xi^{3} \widehat{y}^{3}+\xi^{4} \widehat{z}^{4} \equiv 0 \quad \bmod \xi^{n}
$$

together with the initial conditions $x_{i}=y_{i}=z_{i}=0$ for $i \geqslant n-1$. The twisted initial form is $X^{2}$. According to the algorithm, we have a single branching given by the transformations $e_{X}^{\sharp}$ and $e_{X}$. The twisted initial form of $e_{X}^{\sharp}(1,1,1)=$ $\left(1^{\sharp}, 1,1\right)$ is defined by $X_{\sharp}^{2}=0$ and hence is empty. So remains the untagged leaf $e_{X}(1,1,1)=(2,1,1)$, with jet equations

$$
\xi^{4} \widehat{x}^{2}+\xi^{3} \widehat{y}^{3}+\xi^{4} \widehat{z}^{4} \equiv 0 \quad \bmod \xi^{n}
$$

and in addition to the previous initial conditions, also $x_{n-2}=0$. As the twisted initial form is $Y^{3}$, we branch with $e_{Y}^{\sharp}$ and $e_{Y}$. The twisted initial form of $e_{Y}^{\sharp}(2,1,1)=\left(2,1^{\sharp}, 1\right)$ is $Y_{\sharp}^{3}=0$, whence empty, leaving us with $e_{Y}(2,1,1)=(2,2,1)$, whose jet equations are

$$
\xi^{4} \widehat{x}^{2}+\xi^{6} \widehat{y}^{3}+\xi^{4} \widehat{z}^{4} \equiv 0 \bmod \xi^{n}
$$


and an additional initial condition $y_{n-2}=0$. The new twisted initial form is $X^{2}+Z^{4}$. At this point, inverting either variable $X$ or $Z$ yields a regular surface. However, instead of choosing one, we may perform a multi-branching step, in which we consider all four possibilities $e_{X} e_{Z}, e_{X}^{\sharp} e_{Z}, e_{X} e_{Z}^{\sharp}$, or $e_{X}^{\sharp} e_{Z}^{\sharp}$, when applying (5.9), yielding the four leafs $(3,2,2),\left(2^{\sharp}, 2,2\right),\left(3,2,1^{\sharp}\right)$, and $\left(2^{\sharp}, 2,1^{\sharp}\right)$ respectively. The corresponding initial forms are given by $X^{2}+Y^{3}=$ $0, X_{\sharp}^{2}=0, Z_{\sharp}^{4}=0$, and $X_{\sharp}^{2}+Z_{\sharp}^{4}=0$. The middle two clearly are empty, and as the last is smooth, we may invoke Proposition 5.2, to get

$$
\left[\nabla_{n}^{\left(2^{\sharp}, 2,1^{\sharp}\right)} E_{6}\right]=\left[\tilde{E}_{6}{ }^{\left(2^{\sharp}, 2,1^{\sharp}\right)}\right] \cdot \mathbb{L}^{2 n-2+4-5}=\left[X_{\sharp}^{2}+Z_{\sharp}^{4}\right] \cdot \mathbb{L}^{2 n-3}
$$

as $\operatorname{ord}_{\left(2^{\sharp}, 2,1^{\sharp}\right)}\left(E_{6}\right)=4$. This leaves the first leaf, $(3,2,2)$, with formal jet equations

$$
\xi^{6} \widehat{x}^{2}+\xi^{6} \widehat{y}^{3}+\xi^{8} \widehat{z}^{4} \equiv 0 \bmod \xi^{n}
$$

and the two additional initial conditions $x_{n-3}=z_{n-2}=0$. Its twisted initial form $X^{2}+Y^{3}$ becomes non-singular if we invert $X$ or $Y$, suggesting another multi-branching step. Inverting one and equating the other to zero leads once more to contradictory equations, so we only have to deal with the two leafs $\left(3^{\sharp}, 2^{\sharp}, 2\right)$ and $(4,3,2)$. For the former, we may invoke once more Proposition 5.2 , yielding the class

$$
\left[X_{\sharp}^{2}+Y_{\sharp}^{3}\right] \cdot \mathbb{L}^{2 n-2+6-7},
$$

$\operatorname{as} \operatorname{ord}_{\left(3^{\sharp}, 2,2^{\sharp}\right)}\left(E_{6}\right)=6$. The latter has formal jet equations

$$
\xi^{8} \widehat{x}^{2}+\xi^{9} \widehat{y}^{3}+\xi^{8} \widehat{z}^{4} \equiv 0 \bmod \xi^{n}
$$

together with the vanishing of $x_{i}, y_{i}$ and $z_{i}$ for $i$ greater than or equal to respectively $n-4, n-3$, and $n-2$. Since $(4,3,2)$ has the same twisted initial form as $(2,2,1)$, we may repeat our previous argument. Tagging both variables gives the leaf $\left(4^{\sharp}, 3,2^{\sharp}\right)$ and

$$
\left[X_{\sharp}^{2}+Z_{\sharp}^{4}\right] \cdot \mathbb{L}^{2 n-2+8-9}
$$

as $\operatorname{ord}_{\left(4^{\sharp}, 3,2^{\sharp}\right)}\left(E_{6}\right)=8$. The latter leaf is $(5,3,3)$, with formal jet equations

$$
\xi^{10} \widehat{x}^{2}+\xi^{9} \widehat{y}^{3}+\xi^{12} \widehat{z}^{4} \equiv 0 \bmod \xi^{n}
$$


together with $x_{i}, y_{i}, z_{i}=0$ for $i \geqslant n-5, n-3, n-3$ respectively. As the twisted initial form is $Y^{3}$, we again branch over $e_{Y}^{\sharp}$ and $e_{Y}$ leading to the leaf $(5,4,3)$, with formal jet equations

$$
\xi^{10} \widehat{x}^{2}+\xi^{12} \widehat{y}^{3}+\xi^{12} \widehat{z}^{4} \equiv 0 \quad \bmod \xi^{n}
$$

together with $x_{i}, y_{i}, z_{i}=0$, for $i \geqslant n-5, n-4, n-3$ respectively. As $X^{2}$ is the new twisted initial form, we branch over $e_{X}^{\sharp}$ and $e_{X}$, yielding the leaf $(6,4,3)$, with formal jet equations

$$
\xi^{12} \widehat{x}^{2}+\xi^{12} \widehat{y}^{3}+\xi^{12} \widehat{z}^{4} \equiv 0 \bmod \xi^{n}
$$

together with $x_{i}, y_{i}, z_{i}=0$ for $i \geqslant n-6, n-4, n-3$ respectively. As $W$ itself is the twisted initial form of this leaf, that is to say, $\mathbf{0} \triangleleft_{W}(6,4,3)$, our algorithm has come to a halt. Indeed, if we factor out $\xi^{12}$ in the last equation, we get the $(n-12)$-th formal jet equations. Since we have $|(6,4,3)|=13$ additional initial conditions, we are left with $3 \cdot 12-13=23$ free variables $x_{i}, y_{i}, z_{i}$ for $n-12 \leqslant i<n-6, n-4, n-3$ respectively, as predicted by Lemma 5.3 . Putting everything together, we showed that $\left[\nabla_{n} E_{6}\right]$ is equal to

$$
\left[E_{6}-O\right] \mathbb{L}^{2 n-2}+2\left[\tilde{E}_{6}{ }^{\left(2^{\sharp}, 2,1^{\sharp}\right)}\right] \mathbb{L}^{2 n-3}+\left[\tilde{E}_{6}{ }^{\left(3^{\sharp}, 2^{\sharp}, 2\right)}\right] \mathbb{L}^{2 n-3}+\left[\nabla_{n-12} E_{6}\right] \mathbb{L}^{23}
$$

Multiplying with $t^{n}$, summing over all $n$, and solving for the zeta series yields

$$
\operatorname{Igu}_{E_{6}}=\frac{Q_{E_{6}}}{\left(1-\mathbb{L}^{2} t\right)\left(1-\mathbb{L}^{23} t^{12}\right)}
$$

for some polynomial $Q_{E_{6}}$ over $\mathfrak{G}$. A schematic representation of these calculations is given by the rationalization tree in Figure 2, in which we equated, for brevity, a leaf to the class of the corresponding directed jet scheme (giving only its defining polynomial).

It is now also clear how this generalizes to any diagonal hypersurface, yielding a proof of Corollary 5.10. Indeed, with $e$ the least common multiple of the $a_{i}$, the algorithm stops at the leaf $\gamma:=\left(\frac{e}{a_{1}}, \ldots, \frac{e}{a_{m}}\right)$, whose order is $e$. During this process, we introduced $|\gamma|$ many additional initial conditions. As we have me more jet variables for the $n$-th jet as for the $(n-e)$-th jet, this yields $N=m e-|\gamma|$ free variables, explaining formula (5.12).

Let us apply this algorithm also to the Du Val surfaces $A_{k}$ and $E_{8}$. The former is given by $X^{2}+Y^{2}+Z^{k+1}$. If $k$ is odd, then $e=k+1$ and $N=$ $3(k+1)-\left(\frac{k+1}{2}+\frac{k+1}{2}+1\right)=2 k+1$, and hence

$$
\operatorname{Igu}_{A_{k}}=\frac{Q_{A_{k}}}{\left(1-\mathbb{L}^{2} t\right)\left(1-\mathbb{L}^{2 k+1} t^{k+1}\right)} .
$$




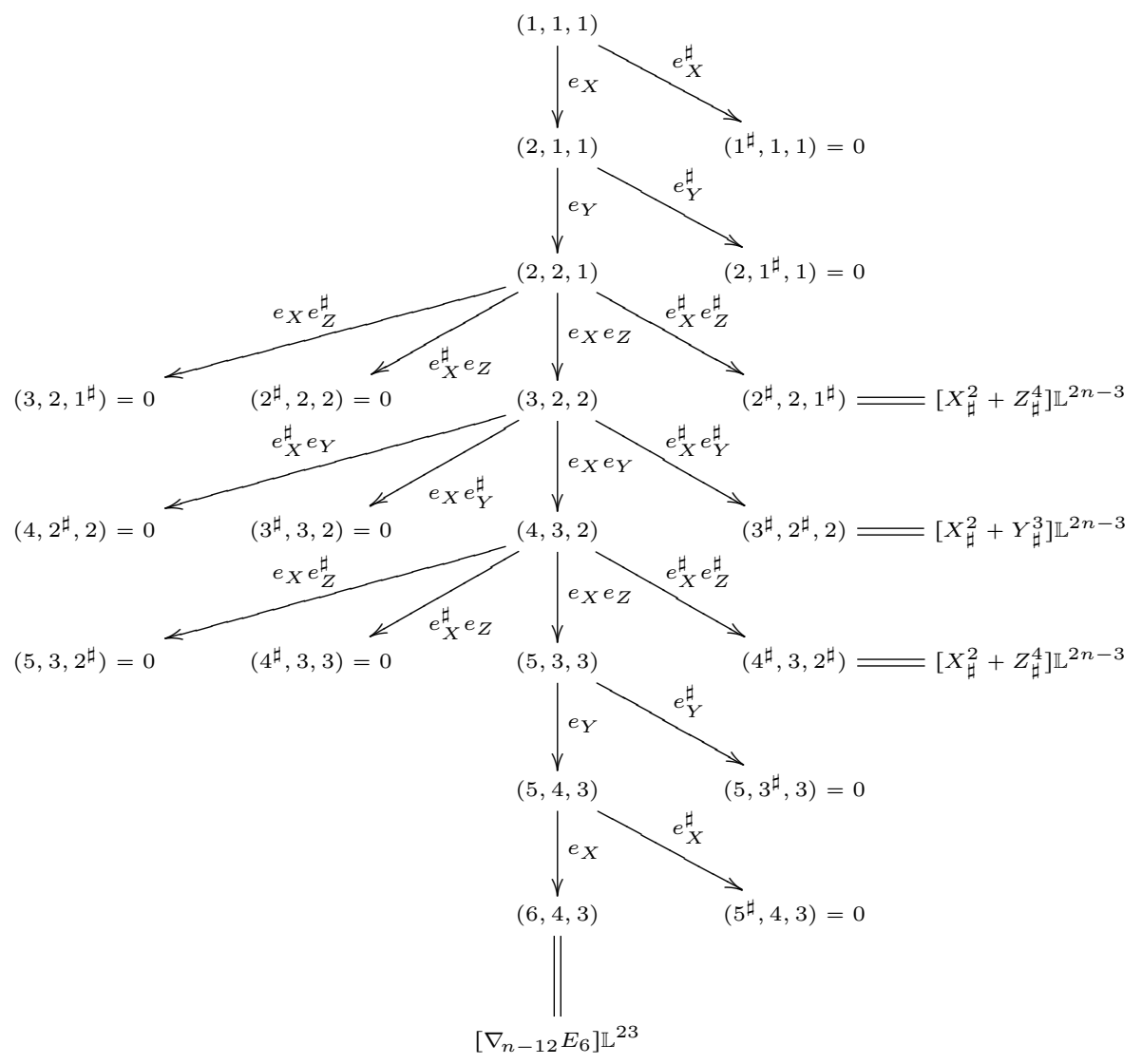

Figure 2. The rationalization tree for the $E_{6}$-surface

If $k$ is even, then $e=2(k+1)$ and $N=6(k+1)-(k+1+k+1+2)=4 k+2$, so that

$$
\operatorname{Igu}_{A_{k}}=\frac{Q_{A_{k}}}{\left(1-\mathbb{L}^{2} t\right)\left(1-\mathbb{L}^{4 k+2} t^{2(k+1)}\right)} .
$$

Finally, since $E_{8}$ has equation $X^{2}+Y^{3}+Z^{5}$, the values are $e=30$ and $N=90-(15+10+5)=60$, so that

$$
\operatorname{Igu}_{E_{8}}=\frac{Q_{E_{8}}}{\left(1-\mathbb{L}^{2} t\right)\left(1-\mathbb{L}^{60} t^{30}\right)} .
$$

Although a priori $\tilde{W}^{\boldsymbol{\theta}}$ depends on the embedding of $W$ in some affine space, its class may be more independent from this embedding. For instance, 
in (6.2), all but the two middle terms are independent from an embedding. To which extent does this hold?

\section{The $E_{7}$-surface}

This time, the defining equation is $X^{2}+Y^{3}+Y Z^{3}=0$ (and, as before, assuming that the characteristic is not equal to 2 or 3 ), which again has an isolated (canonical) singularity. As this is no longer just a sum of powers, it will lead to a more complicated rationalization tree, given in Figure 3 below.

Corollary 5.5 yields

$$
\left[\nabla_{n} E_{7}\right]=q \mathbb{L}^{2 n-3}+\left[\nabla_{n-18} E_{7}\right] \mathbb{L}^{29}
$$

where $q$ is equal to

$\left[E_{7}-O\right] \mathbb{L}+\left[X^{2}+Y Z_{\sharp}^{3}\right]+\left[Y Z_{\sharp}^{3}\right] \mathbb{L}^{-1}+2\left[X_{\sharp}^{2}+Y_{\sharp}^{3}\right]+\left[X_{\sharp}^{2}+Y Z_{\sharp}^{3}\right]+\left[Y_{\sharp}^{3}+Y_{\sharp} Z_{\sharp}^{3}\right]$.

Using the identities

$$
\begin{aligned}
{\left[X^{2}+Y Z_{\sharp}^{3}\right] } & =\mathbb{L}^{2}-\mathbb{L}=\left[Y Z_{\sharp}^{3}\right], \\
{\left[X_{\sharp}^{2}+Y Z_{\sharp}^{3}\right] } & =(\mathbb{L}-1)^{2}, \\
{\left[Y_{\sharp}^{3}+Y_{\sharp} Z_{\sharp}^{3}\right] } & =(\mathbb{L}-1)\left[X_{\sharp}^{2}+Y^{3}\right],
\end{aligned}
$$

we get

$$
q=\mathbb{L}\left[E_{7}-O\right]+(\mathbb{L}+1)\left[X_{\sharp}^{2}+Y_{\sharp}^{3}\right]+2 \mathbb{L}(\mathbb{L}-1) .
$$

Regardless the value of $q$, the usual argument yields the rationality of $\operatorname{Igu}_{E_{7}}$, with denominator equal to $\left(1-\mathbb{L}^{2} t\right)\left(1-\mathbb{L}^{29} t^{18}\right)$.

\section{The $D_{k}$-surface}

The general equation of the $D_{k}$-surface is $X^{2}+Y^{2} Z+Z^{k-1}$ for $k \geqslant 4$ (assuming the characteristic is different from 2). Depending on whether $k$ is odd or even, we have two slightly different rationalization trees, both leading from the root $(1,1,1)$ to $(k-1, k-2,2)$, where

$$
\left[\nabla_{n}^{(k-1, k-2,2)} D_{k}\right]=\left[\nabla_{n-2 k+2} D_{k}\right] \cdot \mathbb{L}^{4 k-5},
$$




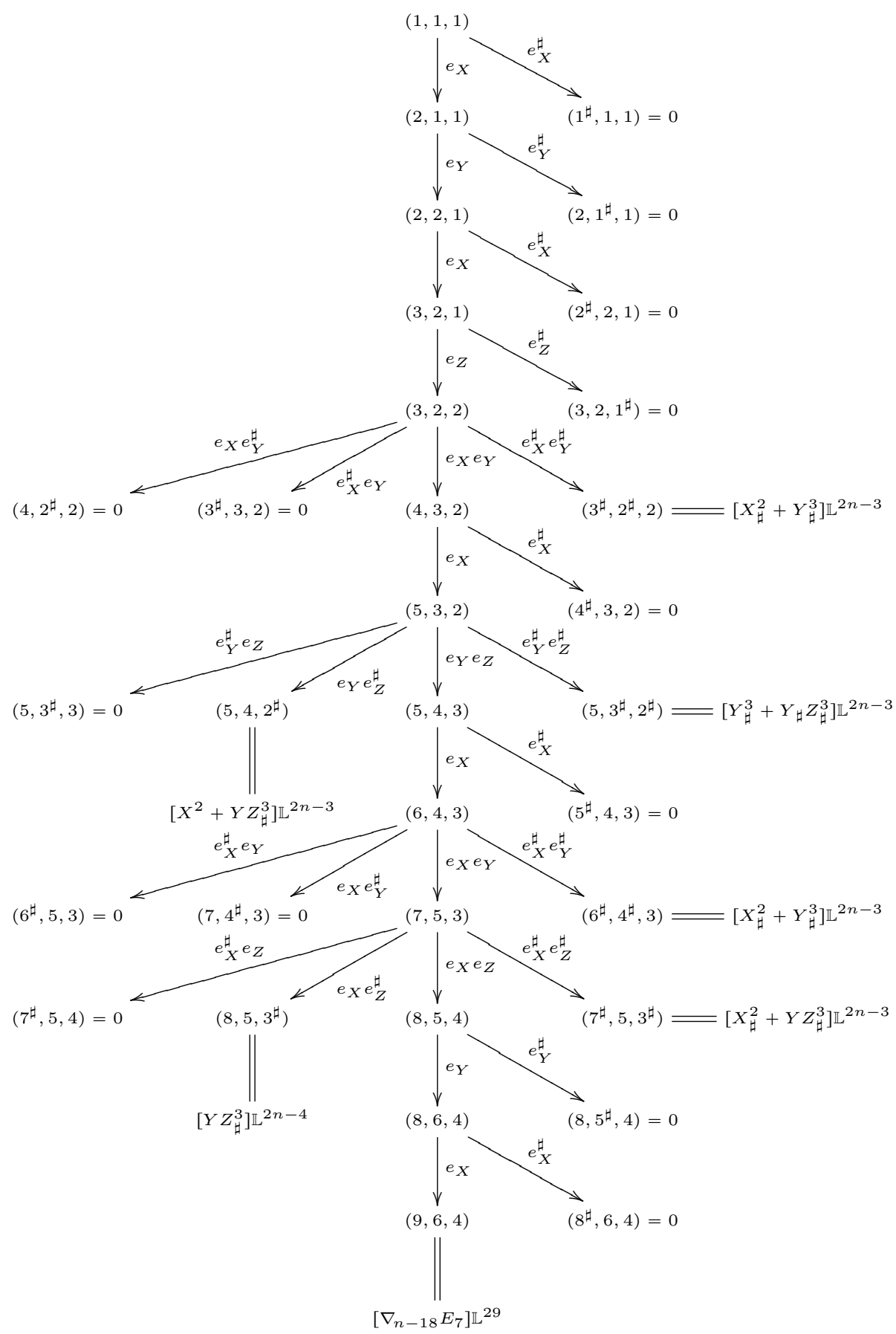

Figure 3 . The rationalization tree for the $E_{7}$-surface 
since we have $3(2 k-2)-|(k-1, k-2,2)|=4 k-5$ free variables. The equations for the directed jet along the starting value $(1,1,1)$ has formal jet equations

$$
\xi^{2} \widehat{x}^{2}+\xi^{3} \widehat{y}^{2} \widehat{z}+\xi^{k-1} \widehat{z}^{k-1} \equiv 0 \bmod \xi^{n}
$$

It is not hard to see that the initial part of the tree is given by alternating $e_{X}$ and $e_{Y}$. As a result, in respectively the first and second term, the power of $\xi$ is each time increased by 2. This goes on until one of them catches up with the power $\xi^{k-1}$, and this depends on the parity of $k$. So assume first that $k$ is odd. In that case, we arrive at the node $\left(\frac{k-1}{2}, \frac{k-1}{2}, 1\right)$, whose directed jet has jet equations

$$
\xi^{k-1} \widehat{x}^{2}+\xi^{k} \widehat{y}^{2} \widehat{z}+\xi^{k-1} \widehat{z}^{k-1} \equiv 0 \bmod \xi^{n}
$$

The remainder of the tree is given in Figure 4 below, where the middle part gets repeated until the indicated value is reached.

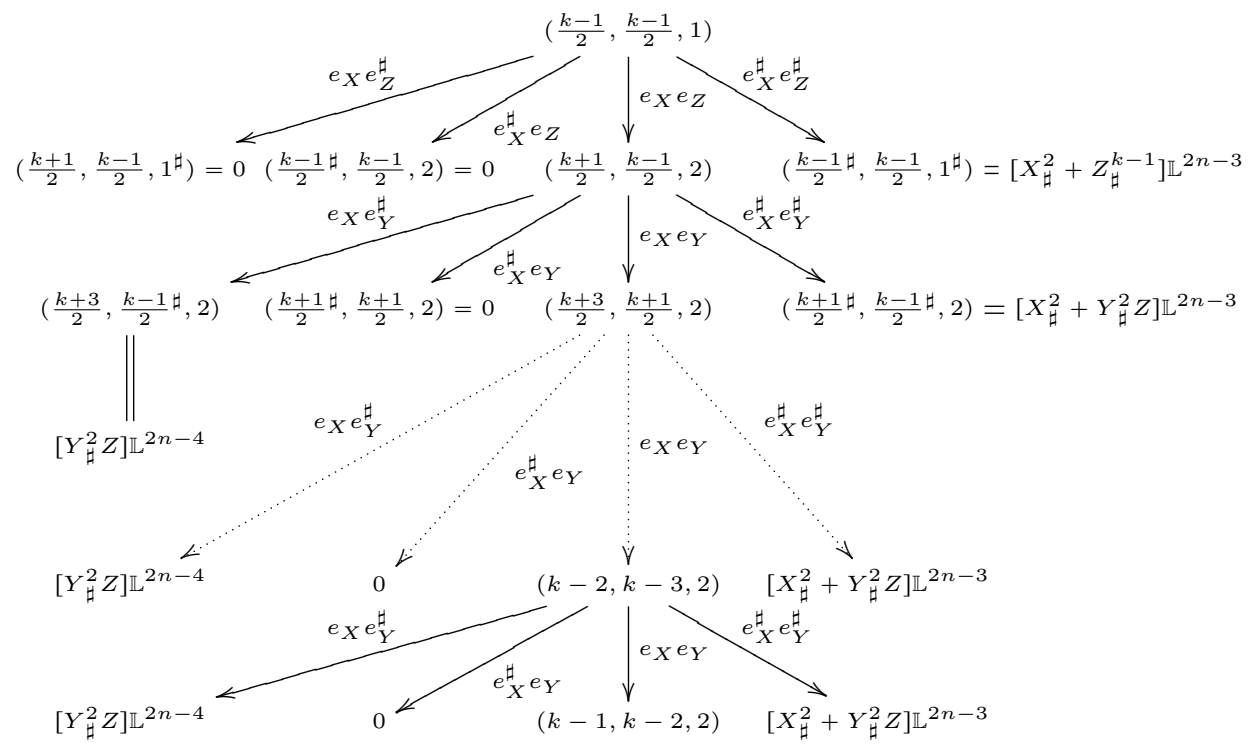

Figure 4. Bottom part of the rationalization tree for $D_{k}$, when $k$ is odd

The tree for even $k$ is analogous, where this time, the starting value is $\left(\frac{k}{2}, \frac{k-2}{2}, 1\right)$, with formal jet equations

$$
\xi^{k} \widehat{x}^{2}+\xi^{k-1} \widehat{y}^{2} \widehat{z}+\xi^{k-1} \widehat{z}^{k-1} \equiv 0 \quad \bmod \xi^{n} \text {. }
$$


The remainder of the tree, with the middle part again repeated, is given by Figure 5 .

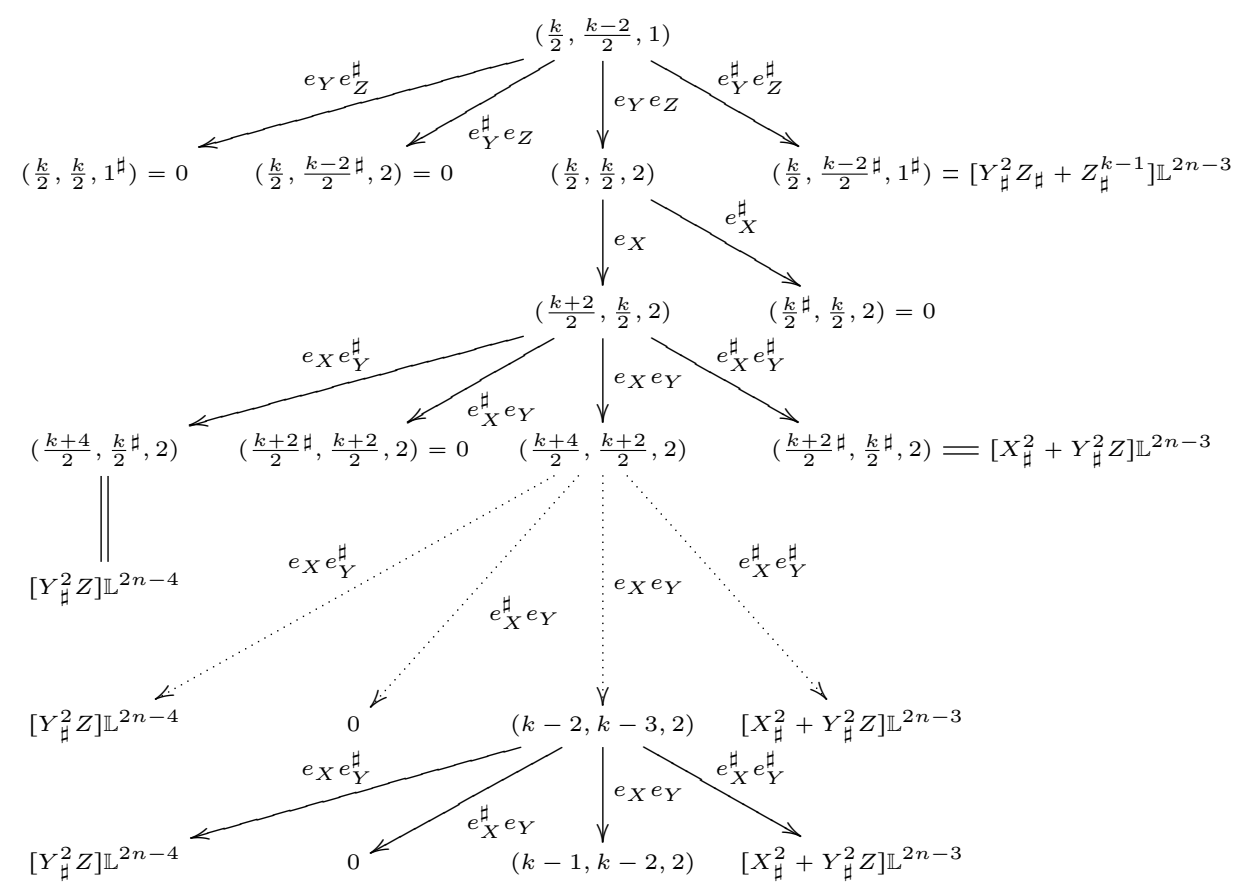

Figure 5. Bottom part of the rationalization tree for $D_{k}$, when $k$ is even

Note that $\left[Y_{\sharp}^{2} Z\right]=\mathbb{L}^{2}-\mathbb{L}$ appears $\left\lceil\frac{k}{2}\right\rceil$ times as an end value, and

$$
\left[X_{\sharp}^{2}+Y_{\sharp}^{2} Z\right]=(\mathbb{L}-1)^{2}
$$

appears $\left\lceil\frac{k+2}{2}\right\rceil$ many times. It follows that $\left[\nabla_{n} D_{k}\right]$ is equal to

$$
\left(\left[D_{k}-O\right] \mathbb{L}+\left[X_{\sharp}^{2}+Z_{\sharp}^{k-1}\right]+\frac{k+1}{2}\left(\mathbb{L}^{2}-\mathbb{L}\right)+(\mathbb{L}-1)^{2}\right) \mathbb{L}^{2 n-3}+\left[\nabla_{n-2 k+2} D_{k}\right] \mathbb{L}^{4 k-5}
$$

in the odd case, and to

$$
\left(\left[D_{k}-O\right] \mathbb{L}+\left[Y_{\sharp}^{2} Z_{\sharp}+Z_{\sharp}^{k-1}\right]+\frac{k}{2}\left(\mathbb{L}^{2}-\mathbb{L}\right)+(\mathbb{L}-1)^{2}\right) \mathbb{L}^{2 n-3}+\left[\nabla_{n-2 k+2} D_{k}\right] \mathbb{L}^{4 k-5}
$$

in the even case. In particular, the motivic Igusa-zeta series $\operatorname{Igu}_{D_{k}}$ is rational with denominator equal to $\left(1-\mathbb{L}^{2} t\right)\left(1-\mathbb{L}^{4 k-5} t^{2 k-2}\right)$. 


\section{Linear deformations of poly-diagonals}

We work over an arbitrary domain $A$. By a poly-diagonal over $A$, we mean a polynomial over $A$ of the form $\sum c_{k} \mathbf{Y}_{k}^{\nu_{k}}$, with $c_{k}$ units in $A$ and $\mathbf{Y}_{k}$ tuples of distinct variables. We define the class of linear deformations of poly-diagonals recursively as follows: 0 belongs to the class, and if $F$ belongs to the class, then so does $F+a X \mathbf{Y}^{\mu}$, where $a$ is a unit in $A, \mu \in \mathbb{N}^{m}$, and $\mathbf{Y}$ an $m$-tuple of variables not yet occurring among the variables of $F$ (but $X$ can be any variable, including one of the $\mathbf{Y}$-variables). A moment's reflection then yields that any linear deformation of a poly-diagonal can be written, for a choice of distinct variables $X_{i}, Y_{i k}, Z_{j}$, in the canonical form

$$
F=H+\sum_{i=1}^{d} a_{i} X_{i}^{n_{i}}+X_{i} G_{i}
$$

with $a_{i}$ units in $A$, and $H(\mathbf{Z})$ (a possibly zero) and the $G_{i}\left(\mathbf{Y}_{i}\right)$ non-zero polydiagonals over $A$. Note that every variable occurs at most once to a higher power. We will refer to the $X_{i}$, that is to say, those variables that occur in at least two terms, as the twisting variables; similarly, we call any monomial occurring in one of the $G_{i}$ a twisted monomial. In other words, if $X \mathbf{Y}^{\nu}$ is a monomial in $F$ with $X$ a twisting variable and $\mathbf{Y}$ a tuple of variables distinct from $X$, then $\mathbf{Y}^{\nu}$ (that is to say, without the twisting variable) is a twisted monomial.

We also introduce the following terminology: we say that a polynomial $F$ is separable in a variable $X$, if either the characteristic is zero, or otherwise, the characteristic is $p>0$ and some exponent of $X$ in $F$ is not divisible by $p$. Put differently, $F$ is non-separable in $X$ if there exists $G$ such that $F=G\left(X^{p}\right)$. We say that $F$ is inseparable, if none of its variables are separable. In particular, over a perfect field, an inseparable polynomial is a $p$-th power. We will say that a linear deformation of a poly-diagonal, written in canonical form (7.1), is almost separable, if no twisted monomial is inseparable and at most one (non-twisted) monomial is inseparable.

Proposition 7.1. If a linear deformation of a poly-diagonal over a field is almost separable, then it is non-degenerated with respect to its Newton polyhedron.

Proof. Let $F$ be an almost separable linear deformation of a poly-diagonal, written in canonical form (7.1). Let $\mathfrak{M}:=\mathfrak{M i l}_{F}$ be its Milnor ideal. Since any subsum in $F$, whence in particular any initial form, is again almost separable, it suffices by Lemma 5.8 to show that $\mathfrak{M}$ contains a (square-free) 
monomial. If $F$ contains a twisted term, the latter must be of the form $X Y^{k} \rho$, with $k$ not divisible by the characteristic, and $\rho$ a monomial in the remaining variables. Since $Y$ only occurs in the twisted term, $\partial F / \partial Y=k X Y^{k-1} \rho$ is a monomial in $\mathfrak{M}$. In the remaining case, $F$ is just a poly-diagonal. By the same argument, if it contains a separable variable, we are done. So we may assume that the characteristic $p$ is prime and $F$ is inseparable, whence by almost separability, a monomial, and so we are done once more.

To give the single branching algorithm that proves the rationality of the motivic Igusa-zeta series of an almost separable linear deformation of a polydiagonal $F$, we describe the two, partially defined, branching functions $\mathcal{A}$ and $\mathcal{A}_{\sharp}$ on $\mathbb{N}_{\sharp}^{m}$, giving the immediate successors of a node in the tree. Write $F$ in the canonical form (7.1), in the $m$ variables $X_{i}, Y_{i j}, Z_{k}$. Renumber the $Y_{i j}$ so that all separable variables are listed first, and do the same with the $Z_{k}$. Let $\mathbf{W}$ be the resulting $m$-tuple of all these variables in the given order, that is to say, starting with the twisting variables $\mathbf{X}$, etc. For $\boldsymbol{\theta} \in \mathbb{N}_{\sharp}^{m}$, let $\mathfrak{M i l}_{\boldsymbol{\theta}}$ be the Milnor ideal of the initial form $\tilde{F}^{\boldsymbol{\theta}}$ viewed as an ideal in $\kappa\left\{\mathbf{W}^{\boldsymbol{\theta}}\right\}$. If $F$ is almost separable, then $\mathfrak{M i l}_{\boldsymbol{\theta}}$ contains at least one (square-free) monomial by Proposition 7.1 .

If $\mathfrak{M i l}_{\boldsymbol{\theta}}$ is the unit ideal, then $\mathcal{A}$ and $\mathcal{A}_{\sharp}$ remain undefined, that is to say, the algorithm halts at such a node, turning it into a leaf. In the remaining case, $\mathfrak{M i l}_{\boldsymbol{\theta}}$ contains a non-zero monomial. Let $\mathcal{S}$ be all monomials in $\mathfrak{M i l}_{\boldsymbol{\theta}}$ none of whose proper factors lie in $\mathfrak{M i l}_{\boldsymbol{\theta}}$. If any of these contain an (untagged) inseparable variable, then we let $V$ be the variable with the highest index in $\mathbf{W}$. If all variables are separable, then we consider only those monomials in $\mathcal{S}$ of minimal length (=minimal number of irreducible factors), and let $V$ be the untagged variable with the highest index among all variables occurring in this latter set of monomials. Define now $\mathcal{A}(\boldsymbol{\theta}):=e_{V} \boldsymbol{\theta}$ and $\mathcal{A}_{\sharp}(\boldsymbol{\theta}):=e_{V}^{\sharp} \boldsymbol{\theta}$ (recall that the former increases the weight of $V$ by one, whereas the latter tags it). Let $\mathcal{T}$ be the (possibly infinite) tree obtained as the orbit of the root 0 under the action of these two branching functions. Note that a node $\boldsymbol{\theta}$ is a leaf precisely when $\mathcal{A}$ (whence also $\mathcal{A}_{\sharp}$ ) is not defined, and such a node is then $F$-regular. In particular, if $\mathcal{T}$ is finite, all its leafs are $F$-regular, and we are done. Therefore, to show that some initial part of $\mathcal{T}$ is rationalizing, we need to show that for each $\boldsymbol{\theta}$ on an infinite branch, we can find $\gamma$ such that $\boldsymbol{\theta} \triangleleft_{F} \boldsymbol{\gamma}$, and we then cut the tree at $\boldsymbol{\gamma}$, turning the latter into a leaf. We induct on the number $m^{\sharp}$ of tagged variables in $\boldsymbol{\theta}$, where there is nothing to prove if $m^{\sharp}=0$, since $\boldsymbol{\theta}$ must then be a leaf. Recall that $\kappa\left\{\mathbf{W}^{\boldsymbol{\theta}}\right\}$ is graded by giving the variables weight $\boldsymbol{\theta}^{\natural}$. We denote the degree of $F$ in this grading by $d(\boldsymbol{\theta}):=\operatorname{deg}_{\boldsymbol{\theta}}(F)$, and its order by $o(\boldsymbol{\theta}):=\operatorname{ord}_{\boldsymbol{\theta}}(F)$.

Lemma 7.2. For $F$ almost separable, with notation as above, if $e$ is the (polynomial) degree of $F$, then $d(\boldsymbol{\theta}) \leqslant o(\boldsymbol{\theta})+e$, for any $\boldsymbol{\theta} \in \mathcal{T}$. 
Proof. We prove this by induction on the height of $\boldsymbol{\theta}$ in $\mathcal{T}$ (that is to say, the number of applications of $\mathcal{A}$ or $\mathcal{A}_{\sharp}$ applied to the root), where the case of the root 0 trivially holds. So assume the result is true for $\boldsymbol{\theta}$ and we have to show it also holds for the next node $\boldsymbol{\theta}^{\prime}=\mathcal{A}(\boldsymbol{\theta})$ (the other node, $\mathcal{A}_{\sharp}(\boldsymbol{\theta})$, has the same $d$ and $o$ values). Let $o:=o(\boldsymbol{\theta})$ and $d:=d(\boldsymbol{\theta})$, so that $d \leqslant o+e$, and write $F=\sum_{i=1}^{s} \mu_{i}$, where the $\mu_{i}$ are the distinct monomials occurring in $F$. Let $V$ be the (necessarily untagged) variable corresponding to the entry that is increased by one in $\boldsymbol{\theta}$. If $V$ is not a twisting variable (that is to say, not one of the $X_{i}$ ), it occurs in a unique monomial $\mu_{i}$, say, as a power $V^{n}$. Moreover, $\mu_{i}$ is then also a monomial in $\tilde{F}^{\boldsymbol{\theta}}$, and $\operatorname{deg}_{\boldsymbol{\theta}}\left(\mu_{i}\right)=o$. Hence $\operatorname{deg}_{\boldsymbol{\theta}^{\prime}}\left(\mu_{i}\right)=o+n$ and since no other monomials have changed their degree, $d\left(\boldsymbol{\theta}^{\prime}\right)$ is the maximum of $d$ and $o+n$, whence by induction at most $o+e$. Since $o \leqslant o\left(\boldsymbol{\theta}^{\prime}\right)$, we showed that $d\left(\boldsymbol{\theta}^{\prime}\right) \leqslant o\left(\boldsymbol{\theta}^{\prime}\right)+e$, as required.

So remains the case that $V$ is a twisting variable. Suppose $V^{n}+V G$ are all terms in $F$ containing $V$, where $G$ is a non-zero poly-diagonal. We claim that $o=n t$, where $t=\operatorname{deg}_{\theta}(V)$. Let $\rho$ be one of the (twisted) monomials in $G$. Assuming the claim, let us show by induction, that the difference (in absolute value) between $\operatorname{deg}_{\boldsymbol{\theta}}(V \rho)$ and $t n$ is at most $e-1$. To this end, let $a:=\operatorname{deg}_{\boldsymbol{\theta}}(V \rho)$. By the claim, $n t=o \leqslant a$, and so, together with our induction hypothesis, we get $0 \leqslant a-t n \leqslant e-1$. Since $\operatorname{deg}_{\boldsymbol{\theta}^{\prime}}\left(V^{n}\right)=t n+n$ and $\operatorname{deg}_{\boldsymbol{\theta}^{\prime}}(V \rho)=a+1$, we see that $1-n \leqslant a+1-t n-n \leqslant e-n$, from which the second claim follows readily. Going back to the main proof, since $o\left(\boldsymbol{\theta}^{\prime}\right) \geqslant o$, it suffices to show that $d\left(\boldsymbol{\theta}^{\prime}\right) \leqslant o+e$. By the first claim, $o=t n$ and so $\operatorname{deg}_{\boldsymbol{\theta}^{\prime}}\left(V^{n}\right) \leqslant t n+n \leqslant o+e$. Moreover, by the second claim, $\operatorname{deg}_{\boldsymbol{\theta}}(V G) \leqslant o+e-1$, and hence $\operatorname{deg}_{\boldsymbol{\theta}^{\prime}}(V G)=\operatorname{deg}_{\boldsymbol{\theta}}(V G)+1 \leqslant o+e$, proving the assertion, as no other terms are involved.

So remains to show the claim. Suppose not, so that $V^{n}$ is not a term in $\tilde{F}^{\boldsymbol{\theta}}$, and let $V \rho_{1}, \ldots, V \rho_{s}$ be all non-zero monomials in $\tilde{F}^{\boldsymbol{\theta}}$ containing $V$. By assumption, no $\rho_{i}$ is inseparable and so the partial derivative with respect to some separable variable proves that each $V \rho_{i}$ lies in $\mathfrak{M i l}_{\boldsymbol{\theta}}$. Therefore, all variables in the $\rho_{i}$ must already be tagged, lest the algorithm would have chosen one of these instead of $V$. If $s=1$, then $\partial \tilde{F}^{\theta} / \partial V=\rho_{1}$ is a unit in $\mathfrak{M i l}_{\boldsymbol{\theta}}$, contradicting that $\boldsymbol{\theta}$ is not $F$-regular (whence a leaf). So remains to show that $s>1$ is also excluded. Suppose not, so that $\operatorname{deg}_{\boldsymbol{\theta}}\left(\rho_{1}\right)=\operatorname{deg}_{\boldsymbol{\theta}}\left(\rho_{2}\right)$ and both monomials $\rho_{i}$ are units in $\kappa\left\{\mathbf{W}^{\boldsymbol{\theta}}\right\}$. Let $\boldsymbol{\gamma}$ be the highest node in the tree below $\boldsymbol{\theta}$ for which not all variables in $\rho_{1}$ or $\rho_{2}$ are tagged. Hence, exactly one variable would not have been tagged, say $U$ in $\rho_{1}=U^{m} \rho_{1}^{\prime}$, with $\rho_{1}^{\prime}$ a unit in $\kappa\left\{\mathbf{W}^{\gamma}\right\}$, and the algorithm at stage $\gamma$ would then pick $U$ and tag it. We must have $\operatorname{deg}_{\gamma}\left(\rho_{1}\right)=\operatorname{deg}_{\gamma}\left(\rho_{2}\right)$, since no stage after $\gamma$ can change their degrees anymore. In particular, $V U^{m} \rho_{1}^{\prime}$ and $\rho_{2}$ are both terms of the initial form $\tilde{F}^{\gamma}$. However, if $T$ is any (necessarily tagged) separable variable in $\rho_{2}$, say, $\rho_{2}=T_{\sharp}^{k} \rho_{2}^{\prime}$, then $\partial \tilde{F}^{\gamma} / \partial T=k V T_{\sharp}^{k-1} \rho_{2}^{\prime}$, showing that $V$ belongs to $\mathfrak{M i l}_{\gamma}$, 
since $k T_{\sharp}^{k-1} \rho_{2}^{\prime}$ is a unit in $\kappa\left\{\mathbf{W}^{\gamma}\right\}$. Since one easily checks that $U V$ but not $U$ belongs to the latter ideal, the algorithm could not have chosen $U$ at that stage since there are monomials with fewer factors in $\mathfrak{M i l}_{\gamma}$, contradiction.

TheOREM 7.3. Let $W \subseteq \mathbb{A}_{\kappa}^{m}$ be a linear deformation of a poly-diagonal with equation $F$. If $F$ is almost separable, then the motivic Igusa zeta series of $W$ is rational.

Proof. By the previous discussion, remains to show, in view of Theorem 5.7, that given any infinite branch of the tree $\mathcal{T}$, as constructed above, we can find two nodes $\gamma_{1}$ and $\gamma_{2}$ on it such that $\gamma_{1} \triangleleft_{F} \gamma_{2}$. To this end, write $F$ as a sum of monomials $\mu_{i}$ and, for each node $\boldsymbol{\theta}$, let $\Omega(\boldsymbol{\theta})$ be the tuple with $i$-th entry equal to $\operatorname{deg}_{\boldsymbol{\theta}}\left(\mu_{i}\right)-o(\boldsymbol{\theta})$ (recall that $o(\boldsymbol{\theta})$ and $d(\boldsymbol{\theta})$ are the respective order and degree of $F$ in $\left.\kappa\left\{\mathbf{Y}^{\boldsymbol{\theta}}\right\}\right)$. By construction all entries in $\Omega(\boldsymbol{\theta})$ are positive, and by Lemma 7.2, at most equal to the degree of $F$. Hence there are only finitely many possibilities for $\Omega(\boldsymbol{\theta})$, so that any infinite branch must contain two nodes $\gamma_{1}<\gamma_{2}$ with $\Omega\left(\gamma_{1}\right)=\Omega\left(\gamma_{2}\right)$. It is now easy to check that the latter is equivalent with $\gamma_{1} \triangleleft_{F} \gamma_{2}$.

\section{References}

[1] Artal Bartolo E., Cassou-Noguès P., Luengo I., Melle Hernández A., Quasi-ordinary power series and their zeta functions, Mem. Amer. Math. Soc. 178 (2005), no. 841, $\mathrm{vi}+85 \mathrm{pp}$.

[2] Bosch S., Lütkebohmert W., Raynaud M., Néron models, Ergebnisse der Mathematik und ihrer Grenzgebiete (3) [Results in Mathematics and Related Areas (3)], vol. 21, Springer-Verlag, Berlin, 1990.

[3] Denef J., Report on Igusa's local zeta function, Séminaire Bourbaki Vol. 1990/91, Astérisque (1991), no. 201-203, Exp. No. 741 (1992), 359-386.

[4] Denef J., Hoornaert K., Newton polyhedra and Igusa's local zeta function, J. Number Theory 89 (2001), no. 1, 31-64.

[5] Denef J., Loeser F., Motivic Igusa zeta functions, J. Algebraic Geom. 7 (1998), no. 3, 505-537.

[6] Denef J., Hoornaert K., Germs of arcs on singular algebraic varieties and motivic integration, Invent. Math. 135 (1999), no. 1, 201-232.

[7] Denef J., Hoornaert K., On some rational generating series occurring in arithmetic geometry, in: Geometric aspects of Dwork theory. Vol. I, II, Walter de Gruyter GmbH \& Co. KG, Berlin, 2004, pp. 509-526.

[8] Eisenbud D., Commutative algebra with a view toward algebraic geometry, Graduate Texts in Mathematics, vol. 150, Springer-Verlag, New York, 1995.

[9] Guibert G., Espaces d'arcs et invariants d'Alexander, Comment. Math. Helv. 77 (2002), no. 4, 783-820.

[10] Hartshorne R., Algebraic geometry, Springer-Verlag, New York, 1977.

[11] Igusa J., A stationary phase formula for p-adic integrals and its applications, in: Algebraic geometry and its applications (West Lafayette, IN, 1990), Springer, New York, 1994, pp. 175-194. 
[12] Igusa J., An introduction to the theory of local zeta functions, AMS/IP Studies in Advanced Mathematics, vol. 14, American Mathematical Society, Providence, RI, 2000.

[13] Lichtin B., Meuser D., Poles of a local zeta function and Newton polygons, Compositio Math. 55 (1985), no. 3, 313-332.

[14] Matsumura H., Commutative ring theory, Cambridge University Press, Cambridge, 1986.

[15] Saia M.J., Zuniga-Galindo W.A., Local zeta function for curves, non-degeneracy conditions and Newton polygons, Trans. Amer. Math. Soc. 357 (2005), no. 1, 59-88.

[16] Schoutens H., Classifying singularities up to analytic extensions of scalars is smooth, Ann. Pure Appl. Logic 162 (2011), 836-852.

[17] Schoutens H., Schemic Grothendieck rings I: motivic sites, Preprint 2011.

[18] Schoutens H., Schemic Grothendieck rings II: jet schemes and motivic integration, Preprint 2011.

[19] Varchenko A., Zeta-function of monodromy and Newton's diagram, Invent. Math. 37 (1976), no. 3, 253-262.

[20] Veys W., Poles of Igusa's local zeta function and monodromy, Bull. Soc. Math. France 121 (1993), no. 4, 545-598.

[21] Veys W., Determination of the poles of the topological zeta function for curves, Manuscripta Math. 87 (1995), no. 4, 435-448.

[22] Zúñiga-Galindo W., Igusa's local zeta functions of semiquasihomogeneous polynomials, Trans. Amer. Math. Soc. 353 (2001), no. 8, 3193-3207.

[23] Zúñiga-Galindo W., Local zeta functions and Newton polyhedra, Nagoya Math. J. 172 (2003), 31-58.

\author{
Department of Mathematics \\ City University of New York \\ 365 Fifth Avenue \\ NEW York, NY 10016 \\ USA \\ e-mail: hschoutens@citytech.cuny.edu
}

